\title{
Tau leptons as a probe for New Physics at LHC
}

\author{
Anna Kaczmarska
}

Institute of Nuclear Physics PAN, Cracow

(On behalf of the ATLAS collaboration)
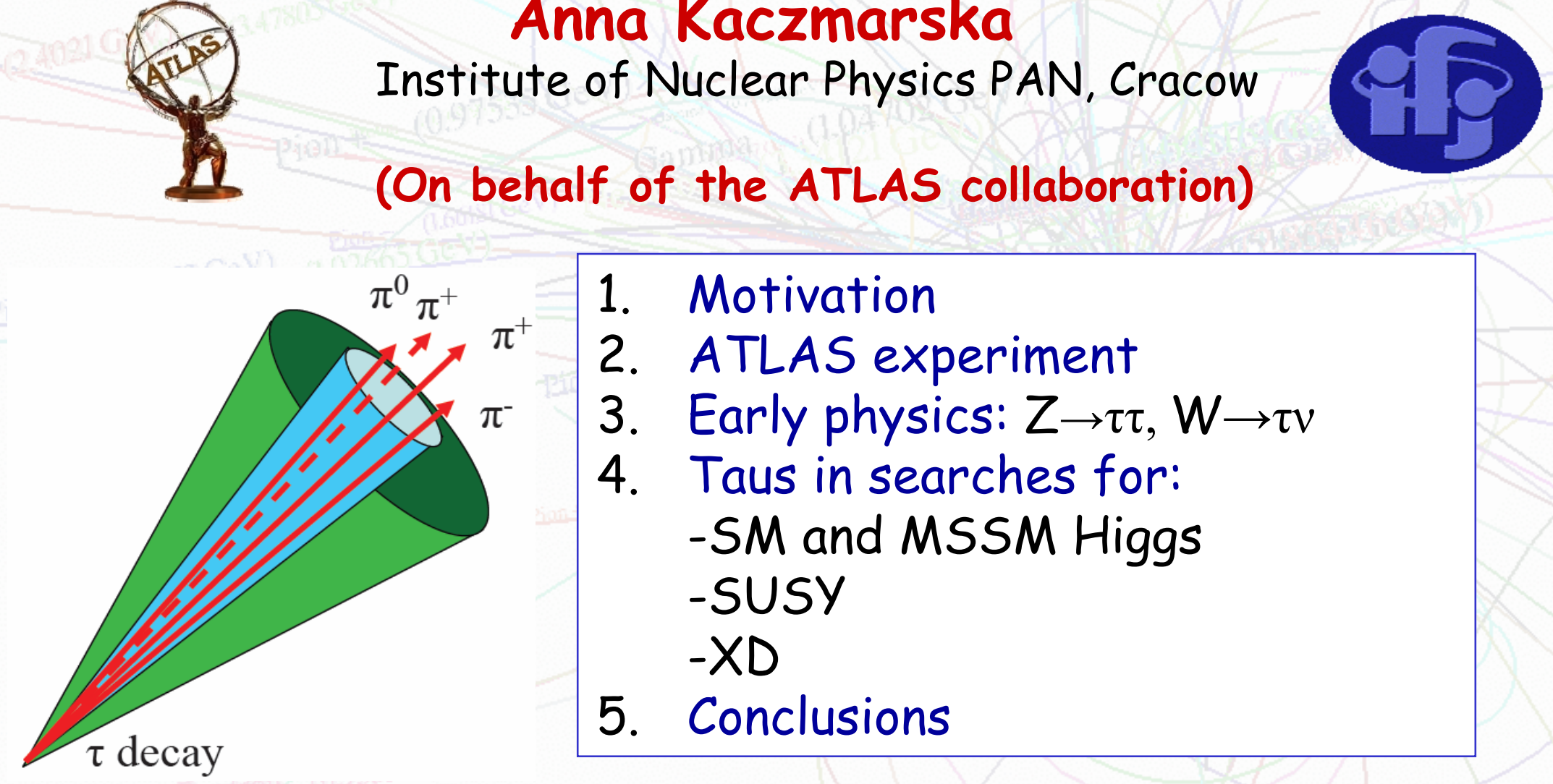

1. Motivation

2. ATLAS experiment

3. Early physics: $Z \rightarrow \tau \tau, W \rightarrow \tau v$

4. Taus in searches for:

-SM and MSSM Higgs

-SUSY

-XD

5. Conclusions 


\section{Why tau leptons are an important signature at LHC experiments}

\section{Taus}

- massive particles, EW interaction only - Yukava coupling to SUSY particles, free from $Q C D$ effects

- production and decay well separated in time, potential for measurement of the polarisation, spin correlations, parity - excellent knowledge about decay modes from low-energy experiments

Ideal signature to probe "New Physics" however .....

- several decay modes possible

- jet-like signature

- difficult because of huge QCD bkg

Make it quite difficult for observing in the pp collision experimental environment

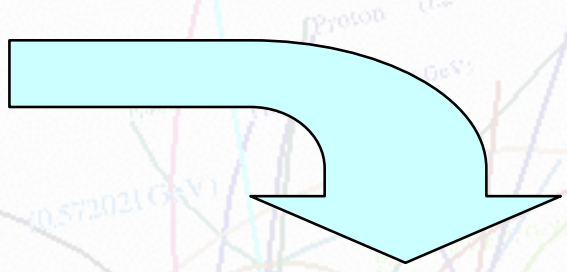

At the LHC

- Large statistics already in the first data: $W \rightarrow \tau v, Z \rightarrow \tau \tau$

- detector calibration, bkg normalisation, algorithms tuning, control channels

$\Rightarrow$ Signature for Higgs boson(s) discovery

- Signature for SUSY discovery

- Polarisation sensitive to SUSY parameters

- Signature for "extra dimensions" 


\section{ATLAS (A Toroidal LHC ApparatuS)}

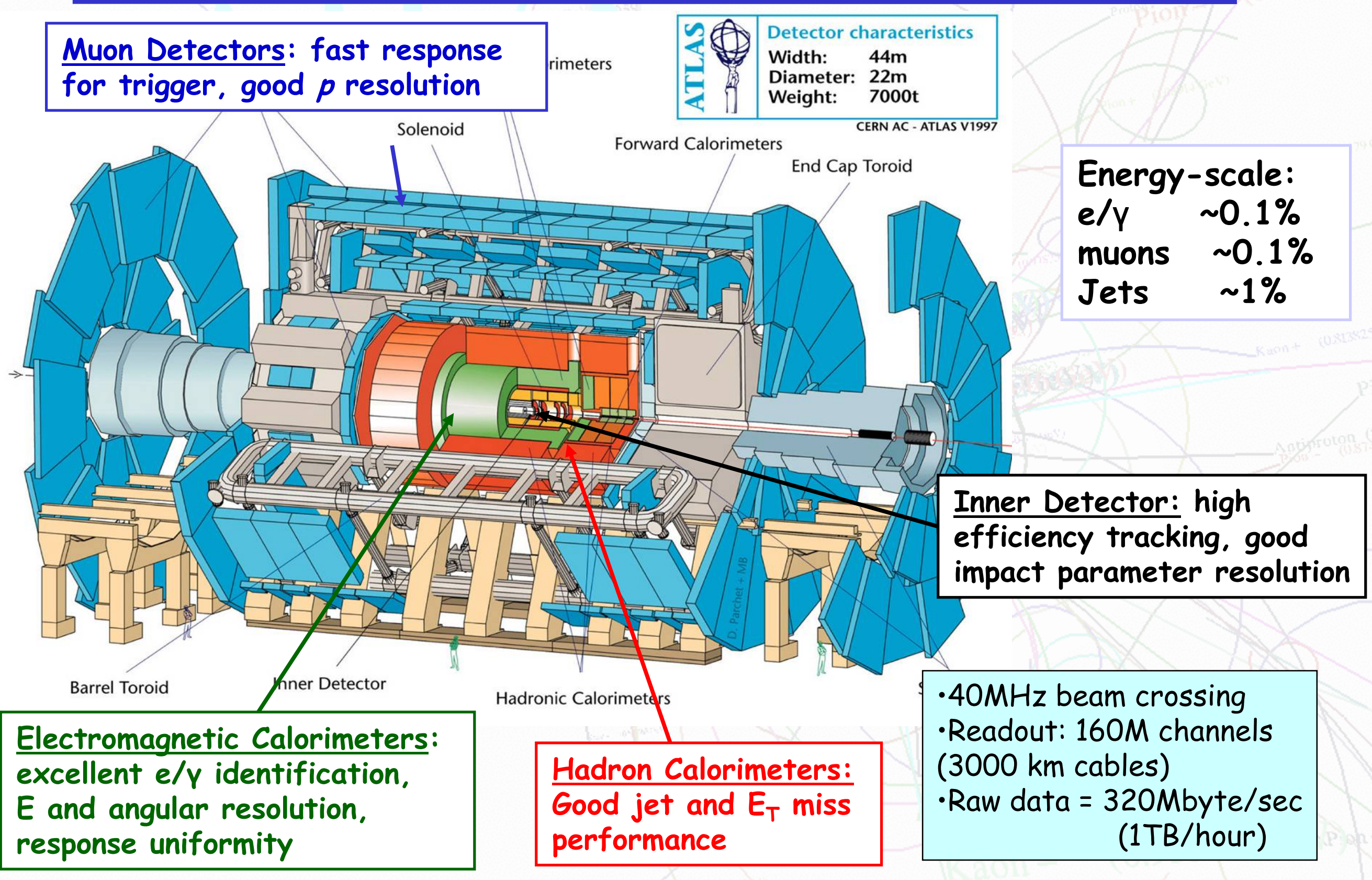

Anna Kaczmarska

Tau 06, Pisa, September 2006 


\section{Machine start up scenario}
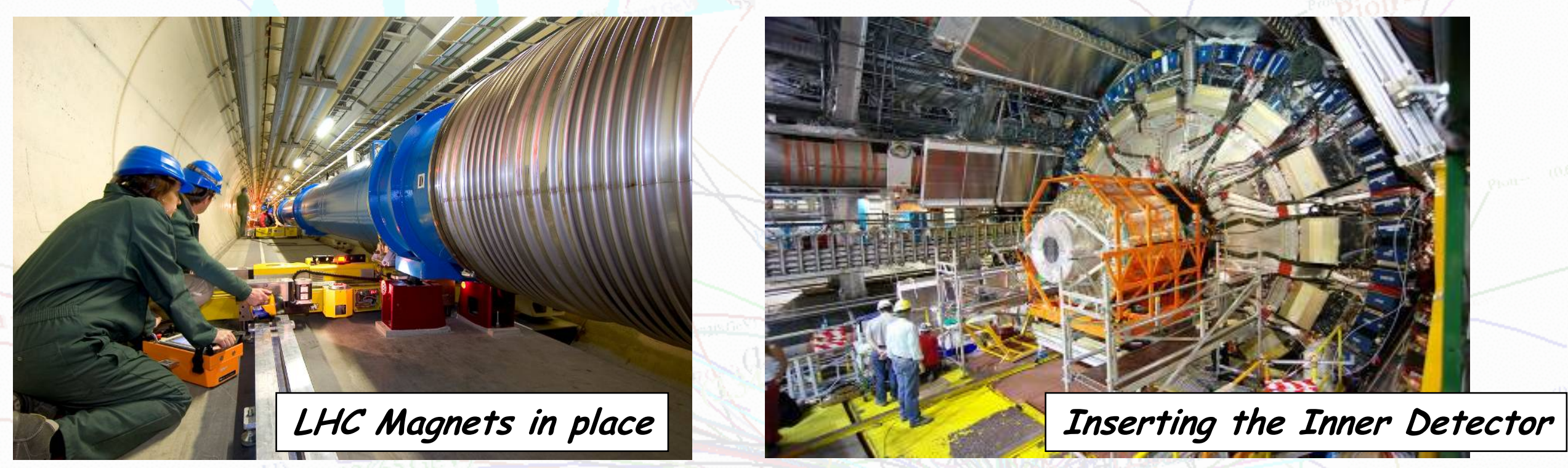

March 2007

$\sim$ August 2007

November 2007
- last LHC magnet installed

- machine and experiments closed

- first collisions $\left(\sqrt{s}=900 \mathrm{GeV}, \mathrm{L} \sim 10^{29} \mathrm{~cm}^{-2} \mathrm{~s}^{-1}\right)$

Commissioning run at injection energy until end 2007 , then shutdown (3 months ?) June 2008 - first collisions at $\sqrt{ } s=14 \mathrm{TeV}$ (followed by first physics run), L 1032 $\mathrm{cm}^{-2} \mathrm{~s}^{-1}$

Goal : deliver integrated luminosity of few fb-1 by end 2008 (with $50 \%$ efficiency of data taking) 


\section{First data: $W \rightarrow \tau v$ events}

Prospects for taus with 10-100 $\mathrm{pb}^{-1}$ at $L \sim 10^{31-32}$ over the first weeks of running: extract signal from most abundant sources of $\tau$ leptons as early as possible $\rightarrow$ requires a performant $\tau$ and $E_{T}$ miss trigger from the very start

\section{Events exp in $100 \mathrm{pb}^{-1}$ vs $\mathrm{N}$ Tau tracks}

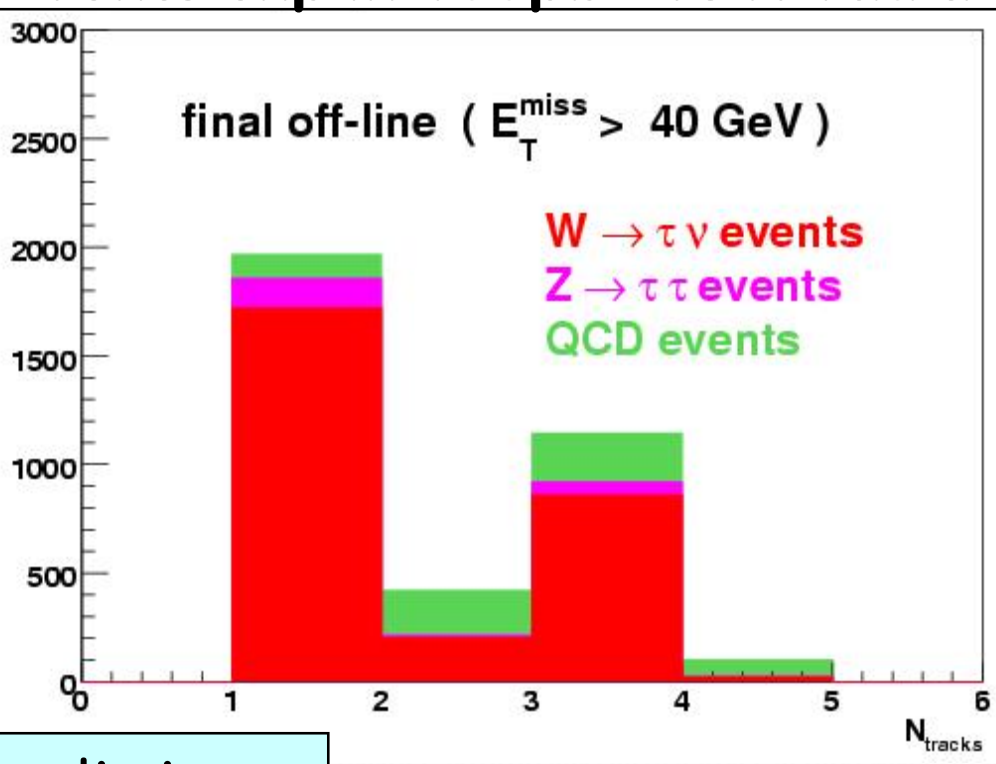

preliminary

\begin{tabular}{|c|c|c|c|}
\hline $\begin{array}{c}\text { Expected rates } \\
\text { for } 100 \mathrm{pb}^{-1}\end{array}$ & $\begin{array}{c}\mathrm{W} \rightarrow \tau \mathrm{v}, \\
\tau \rightarrow \text { hadron }\end{array}$ & $\mathrm{W} \rightarrow \mathrm{ev}$ & $\begin{array}{c}\mathrm{Z} \rightarrow \tau \tau, \\
1 \tau \rightarrow \text { hadron }\end{array}$ \\
\hline$\sigma . \mathrm{B}(\mathrm{pb})$ & 11200 & 17300 & 1500 \\
\hline$\tau 30 \mathrm{i}+\mathrm{xE} 35$ & $\sim 15000$ & $\sim 250000$ & $\sim 1300$ \\
\hline$\tau 20 \mathrm{i}+\mathrm{xE} 25$ & $\sim 60000$ & $\sim 560000$ & $\sim 3500$ \\
\hline
\end{tabular}

Assuming eff $\sim 80 \%$ for $\tau$ trigger, $\sim 50 \%$ for $\tau$ recolid

It will be "counting" experiment: evidence in the $N_{\text {Track }}$ spectrum. Signal $\times 10$ and bgd $\times 100$ with respect to $2 \mathrm{TeV}$ collisions. Profit from low-luminosity operation to trigger at lowest possible thresholds $\left(E_{T} \tau 15 i\right)$, raise $E_{T}$ miss cut as luminosity goes up.

Require QCD jet rejection of $10^{3}-10^{4}$ at $50 \%$ efficiency and $p_{T} \sim 20 \mathrm{GeV}$ 


\section{First data: $Z \rightarrow \tau \tau$ events}

Observation of Z-> $\tau \tau$ events will be "easier", but $10 x$ less events produced

$\rightarrow$ trigger on lepton (electron, muon)

$\rightarrow$ use same-sign (lep,tau) events to control bgd for the signal events, which are opposite-sign

$\rightarrow$ evidence in Ntrack spectrum and Mvis (lep-had) system

$\rightarrow$ reconstruct invariant mass of the $\tau \tau$ system (collinear approximation)

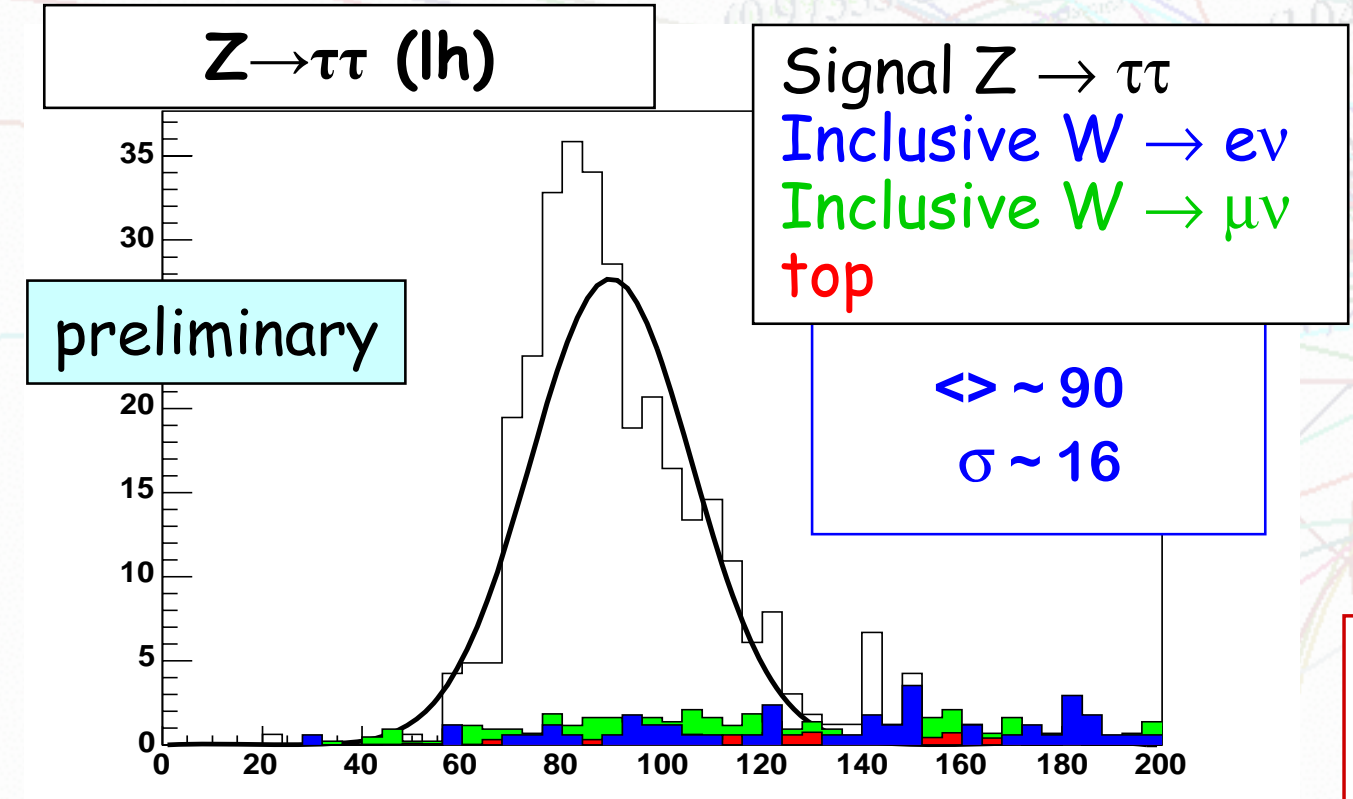

Expect in $100 \mathrm{pb}^{-1}$ about 300 evt observed $(e, \mu)$ with $20 \%$ bgd possibility to loosen cuts? bb bkg still to be included/checked
With $10^{31}$ luminosity, lower threshold on lepton and tau to $15 \mathrm{GeV}$. Tighten selection to improve resolution of invariant mass.
Sensitivity of the measured Z-mass to the absolute energy scale on reconstructed missing energy: $\pm 10 \%$ variation on $E_{T}$ miss results in shift of about $3 \%$ of the measured mass 


\section{Prospects for discovery of Standard Model Higgs boson}

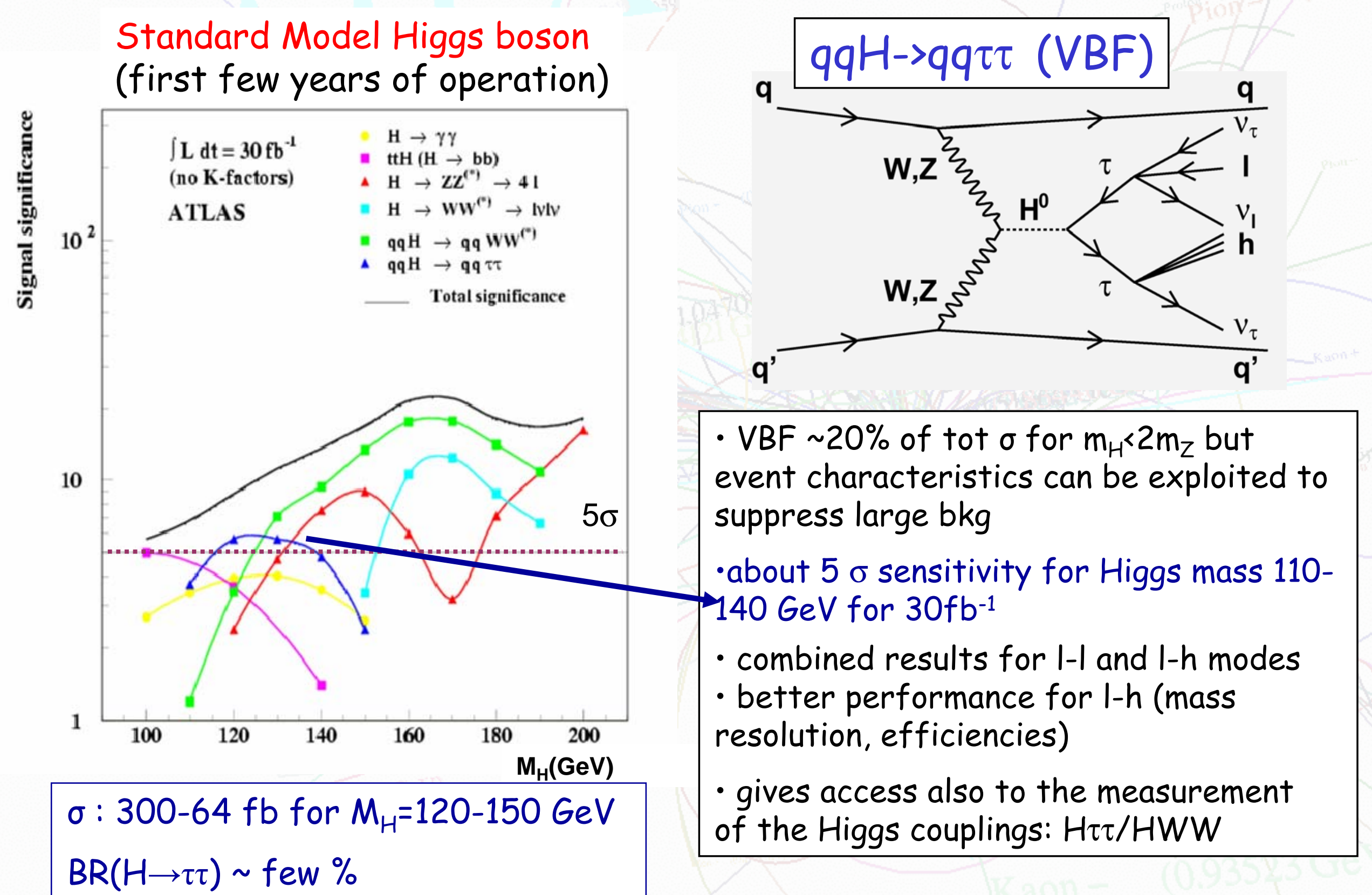




\section{Vector Boson Fusion: $H \rightarrow \tau \tau$}

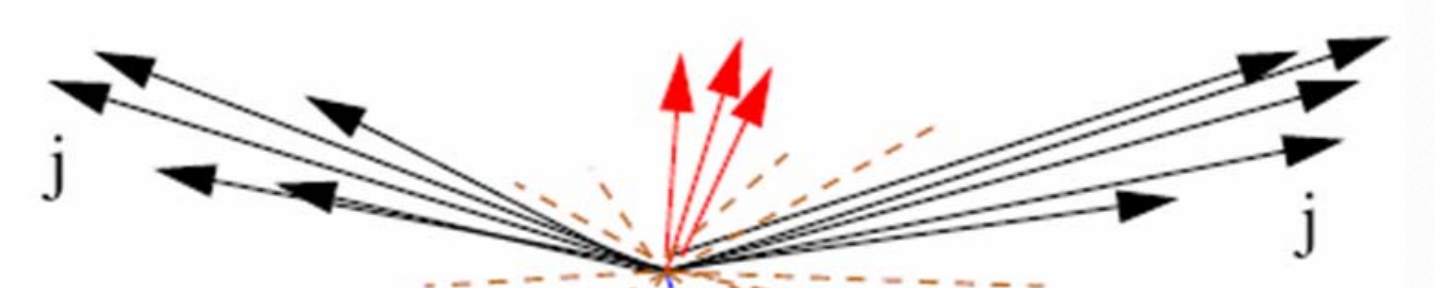

Central Jet Veto:

-Sensible to PileUp

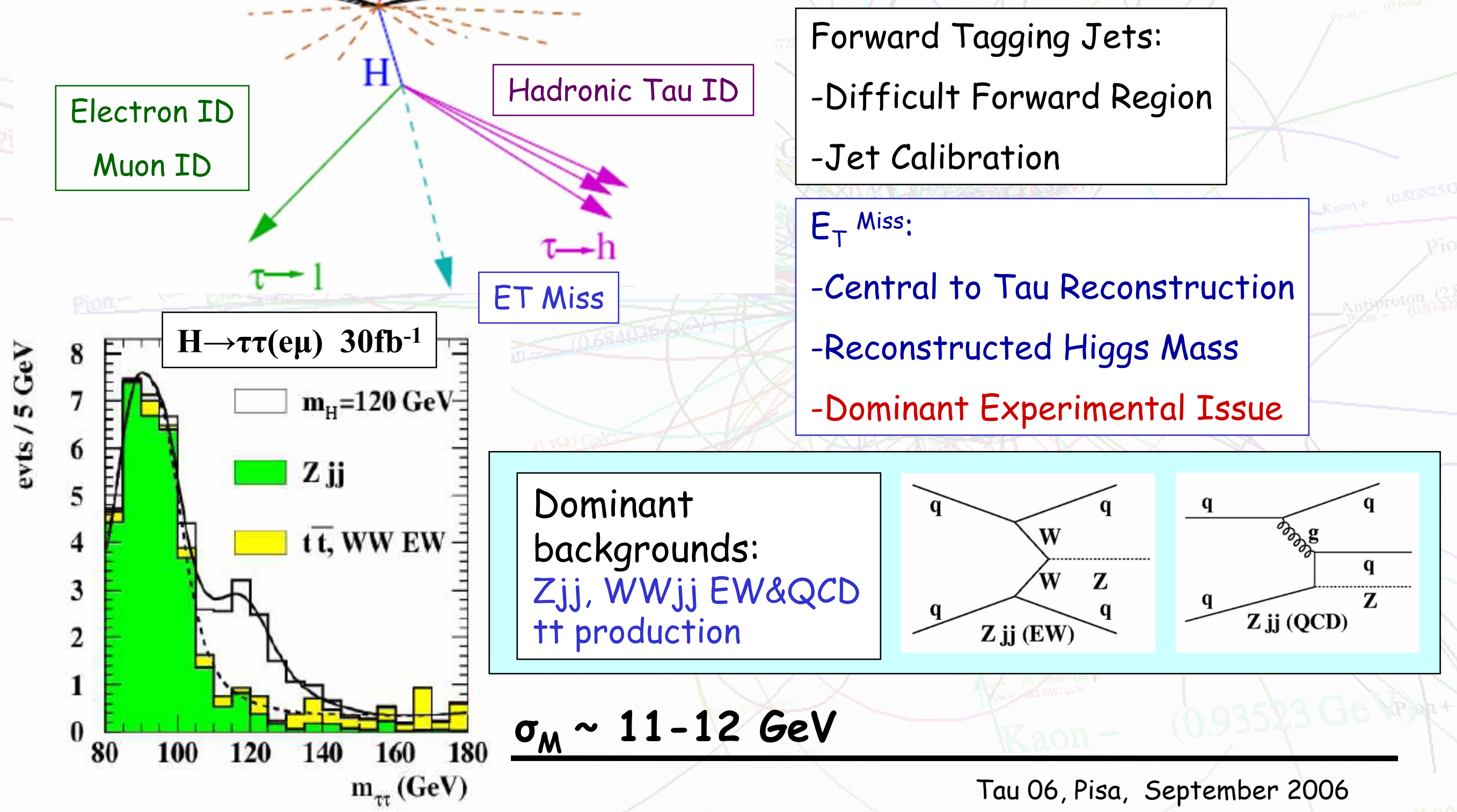




\section{Prospects for discovery of MSSM Higgs boson}

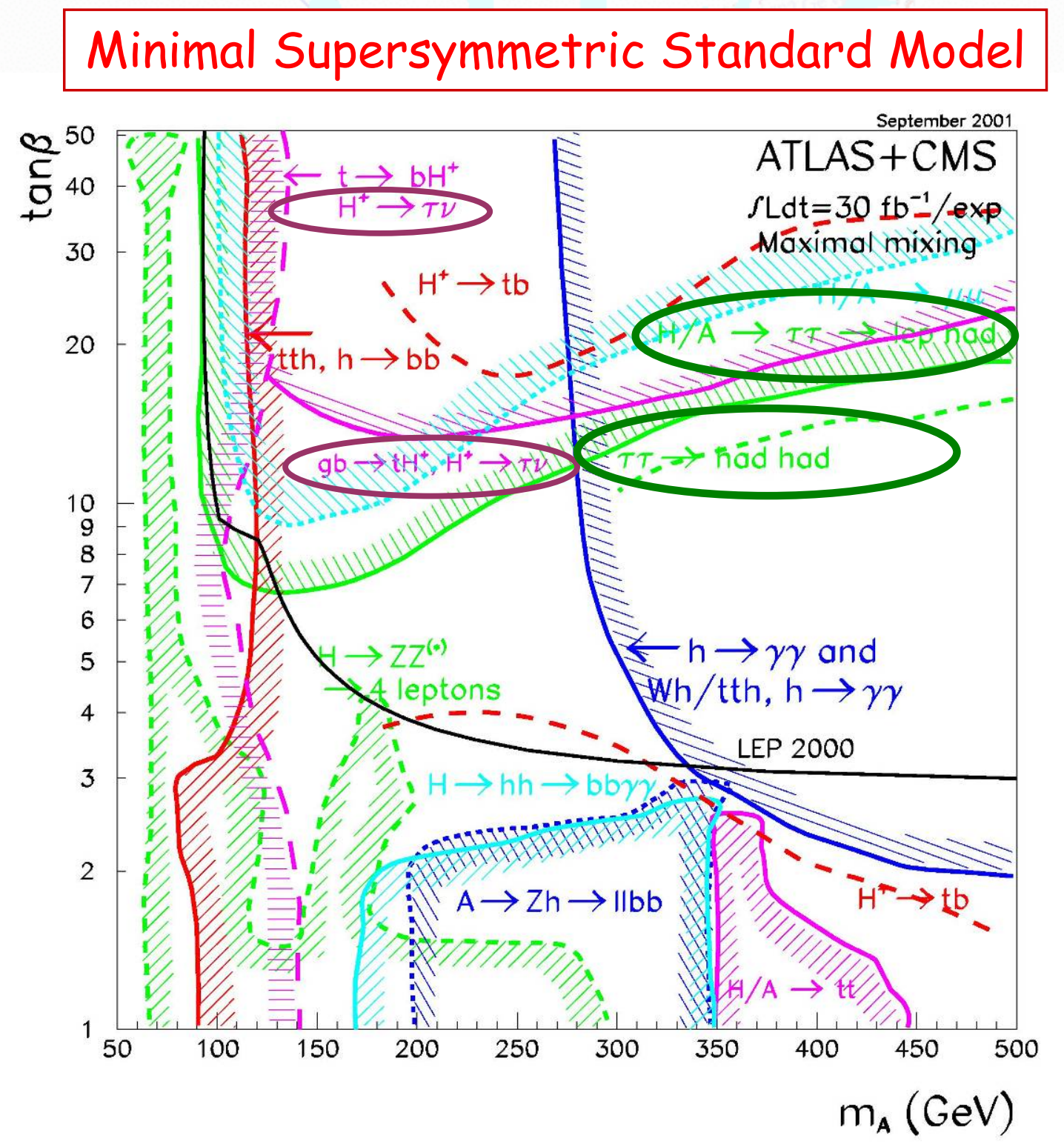

Neutral $H$ and $A$

- prod: $g g->H / A$ and $g g->b b H / A$ (for high $\tan \beta$ )

- $B R(H / A->\tau \tau) \sim 10 \%$

- $B R(H / A->b b) \sim 90 \%$

Charged Higgs -prod:

$m_{H^{\prime}}<m_{+}: \dagger \dagger->W b$ b H $H^{+-}$ $m+>m+: g b \rightarrow+H^{+-}, g g(q q) \rightarrow+b H^{+-}$, $q 9 \rightarrow \mathrm{H}^{+-}$

-decay modes: $\mathrm{H}^{+-}>\tau v, \mathrm{H}^{+-}->$tb for $m_{H}>m_{+}$

$-B R\left(H^{ \pm} \rightarrow \tau v\right) \sim 100 \%$ below $t b$ kinematical threshold

$\rightarrow$ discovery modes for large and moderate $\tan \beta$

$\rightarrow$ measurement of $\tan \beta$ 


\section{Closer look at neutral $H / A \rightarrow \tau \tau$}

The tau decays provide the cleanest signature for the heavy Higgs discovery at high mass (and relatively high $\tan \beta$ )

Investigated all the final states (II, Ih, hh). All of them contribute (at different $M_{A}$ ).

The associated production ( $b b A / H)$ provides additional rejection against the main backgrounds: Z+jet, W+jet, QCD (h-h only)

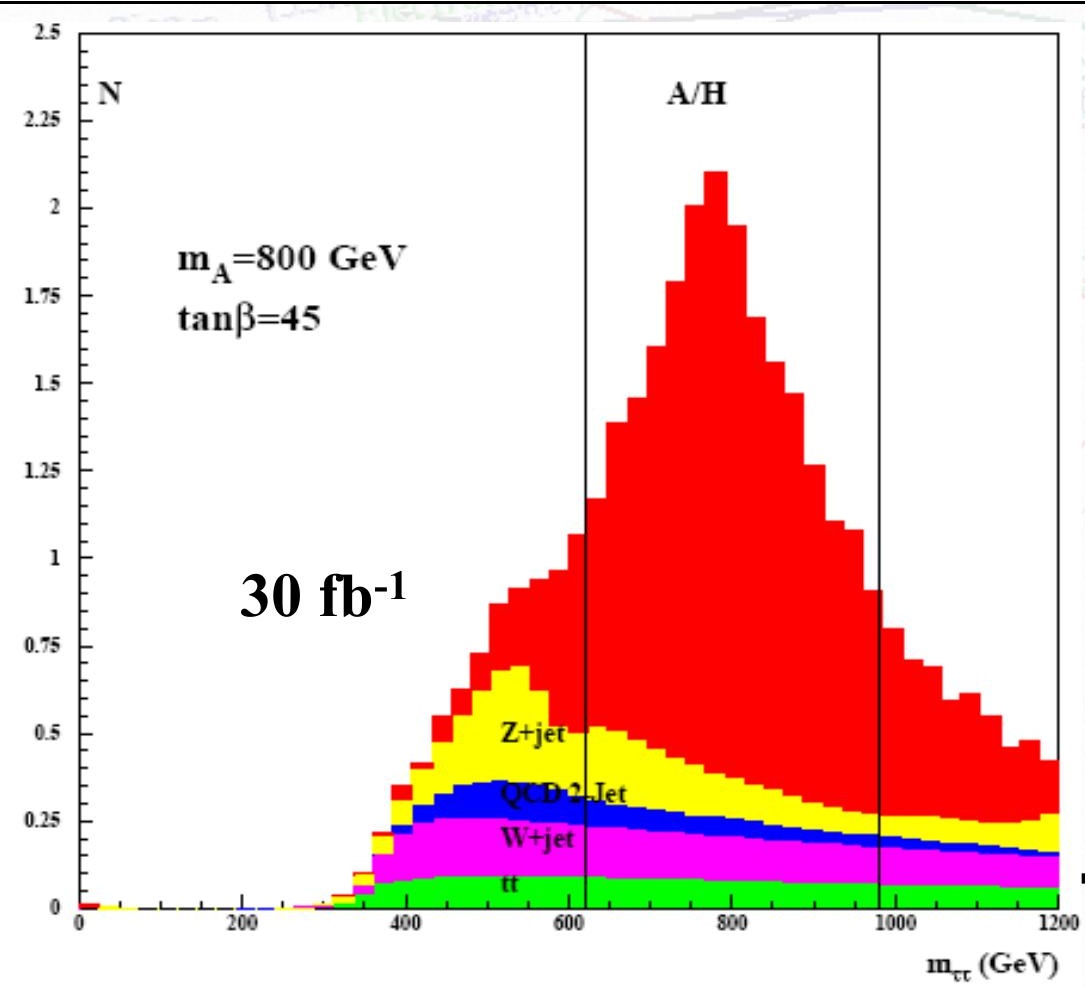

mass resolution: below $15 \%$ in had-had mode

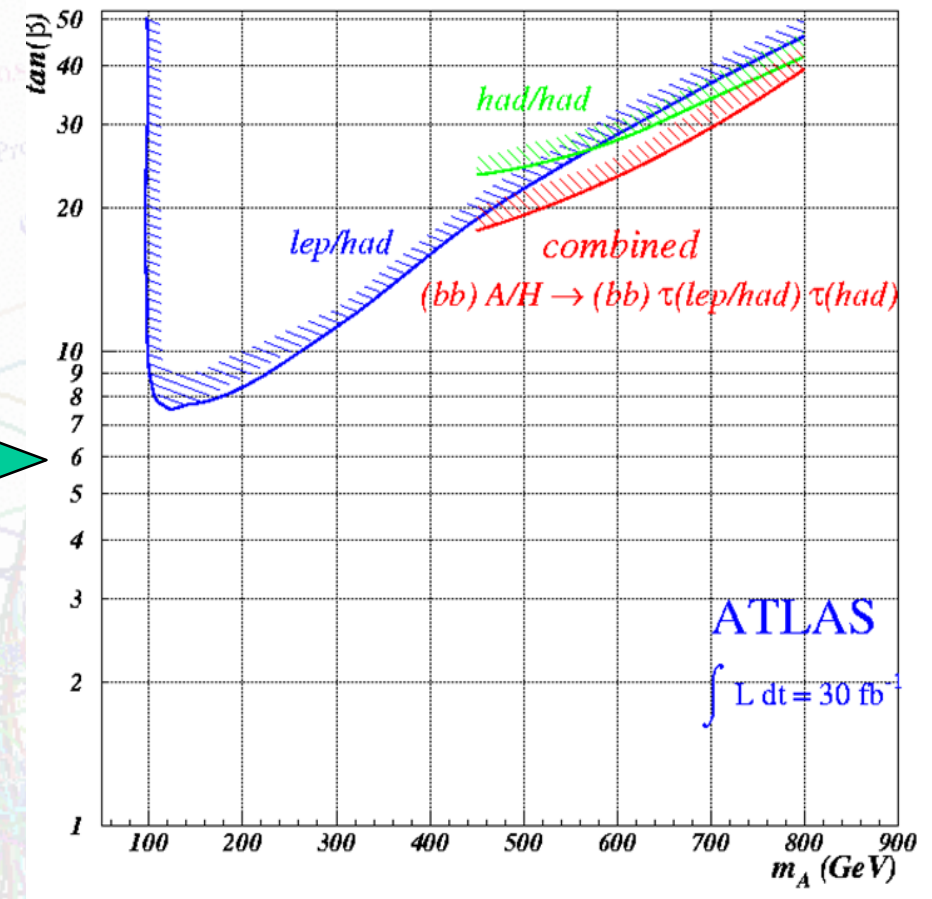

Backgrounds:

$I-h:$ W+jets, Z+jets, tt, bb

$h-h: W+j e t s, Z+j e t s$, tt and QCD

the dominant bgd changes depending on the $m A$ 


\section{Closer look at charged $\mathrm{H}^{ \pm} \rightarrow \tau \nu$}

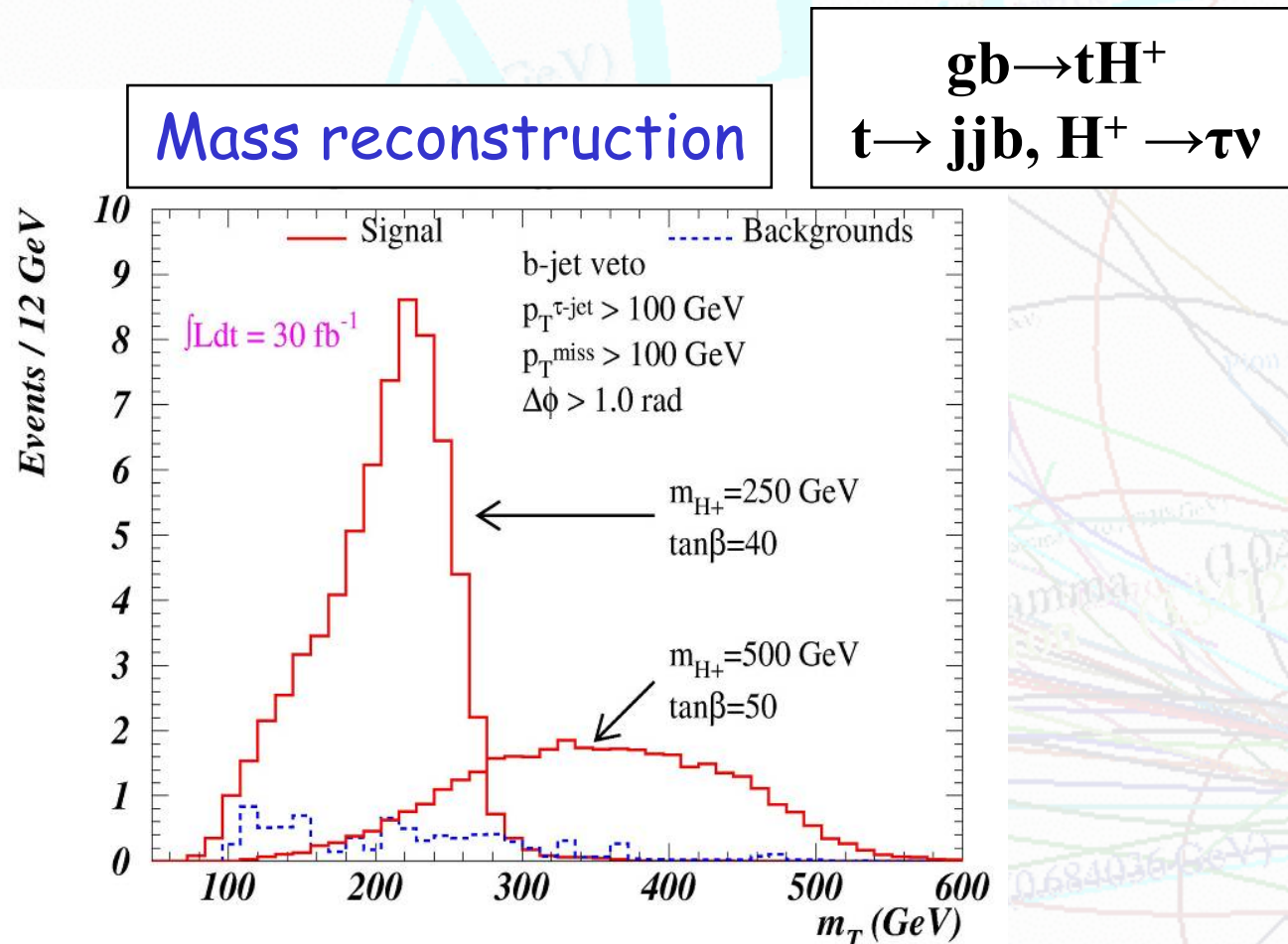

$\rightarrow$ final state: hadronic tau, 3 jets, $E_{T}$ miss

$\rightarrow$ only transverse mass can be reconstructed (as $v$ in final state) but $m_{H}$ can be obtained with likelihood method

$\rightarrow$ almost bgd free channel $(t+, W t)$

$\rightarrow 100 \%$ tau polarisation enhances signal observability above bkg from $W$ events

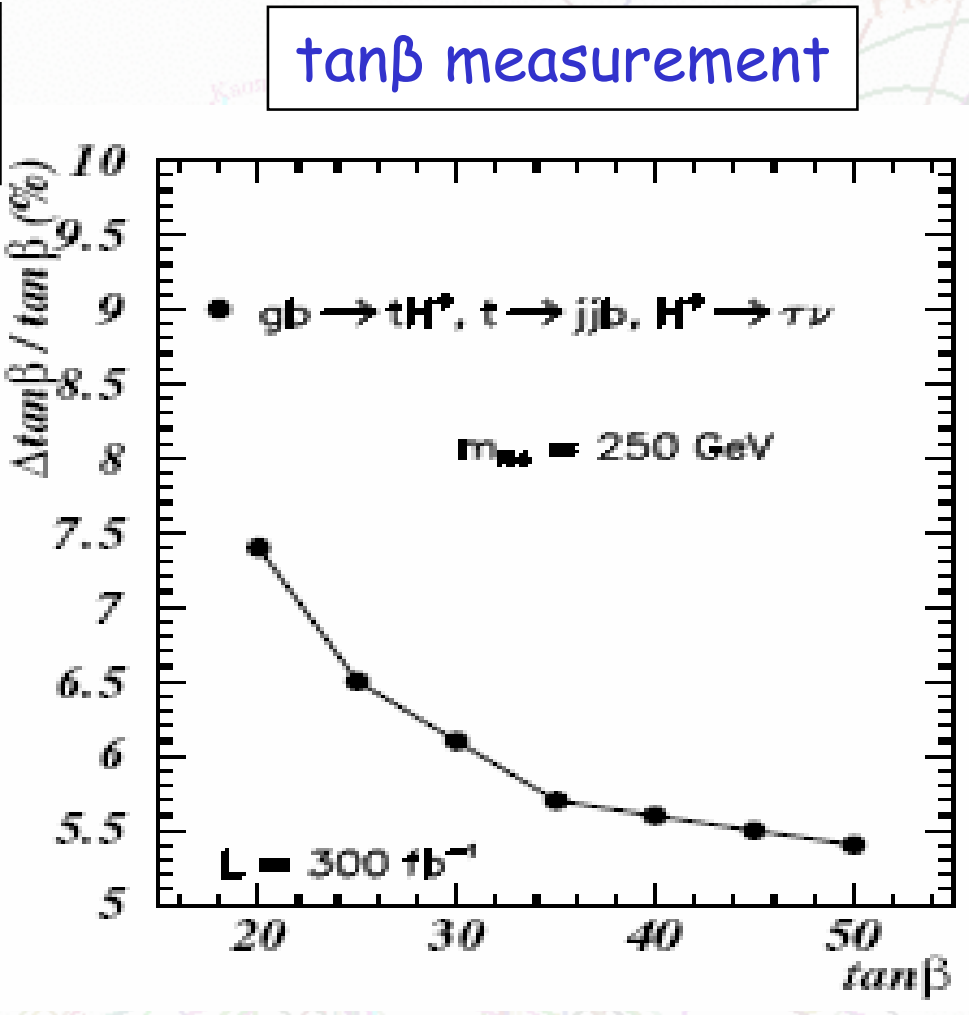

$\rightarrow$ determination of $\tan \beta$ by measuring of the rate in this channel

$$
\sigma\left(\mathrm{gb} \rightarrow \mathrm{tH}^{+-}\right) \times \mathrm{BR}\left(\mathrm{H}^{+-} \rightarrow \tau \nu\right) \sim \tan ^{2} \beta
$$

$\rightarrow$ precision improves as the rate of $\mathrm{H}^{+-} \rightarrow \tau v$ improves with $\tan \beta$ 


\section{If large "extra dimensions" exist .....?}

- In 2-Higgs Doublet Model of type II, 2HDM-II (MSSM)

$$
H^{-} \rightarrow \tau_{R}^{-} \bar{\nu}, \quad H^{-} \text {to } \tau_{L}^{-} \text {suppressed }
$$

- In Large Extra Dimensions $H^{-} \rightarrow \tau_{L}^{-} \psi$ can be enhanced by large number of Kaluza-Klein states. Thus:

$$
H^{-} \rightarrow \tau_{R}^{-} \bar{\nu}+\tau_{L}\left(\psi-\frac{\text { Bulk neutrino }}{\mathrm{M}_{2}=21}\right.
$$

$M_{\mathbf{t}}=210^{4} \mathrm{GeV}, \delta=3, m_{t}=145 \mathrm{eV}, m_{\mathbf{H}}=365.4 \mathrm{GeV}, \tan \beta=45$

- Measurement of the polarisation asymmetry can be used (A func (model parameters))

$$
A=\frac{\Gamma\left(H^{-} \rightarrow \tau_{L}^{-} \psi\right)-\Gamma\left(H^{-} \rightarrow \tau_{R}^{-} \bar{\nu}\right)}{\Gamma\left(H^{-} \rightarrow \tau_{L}^{-} \psi\right)+\Gamma\left(H^{-} \rightarrow \tau_{R}^{-} \bar{\nu}\right.}
$$

- Observation of signal in $m_{T}$ distribution is not sufficient to distinguish between 2 HDM and L.E.D.

- The reconstruction of $p_{\pi} / E_{\text {jet }}$ should determine the scenario: 2HDM or L.E.D.

- Further measurement of the asymmetry may provide a distinctive signature for L.E.D.

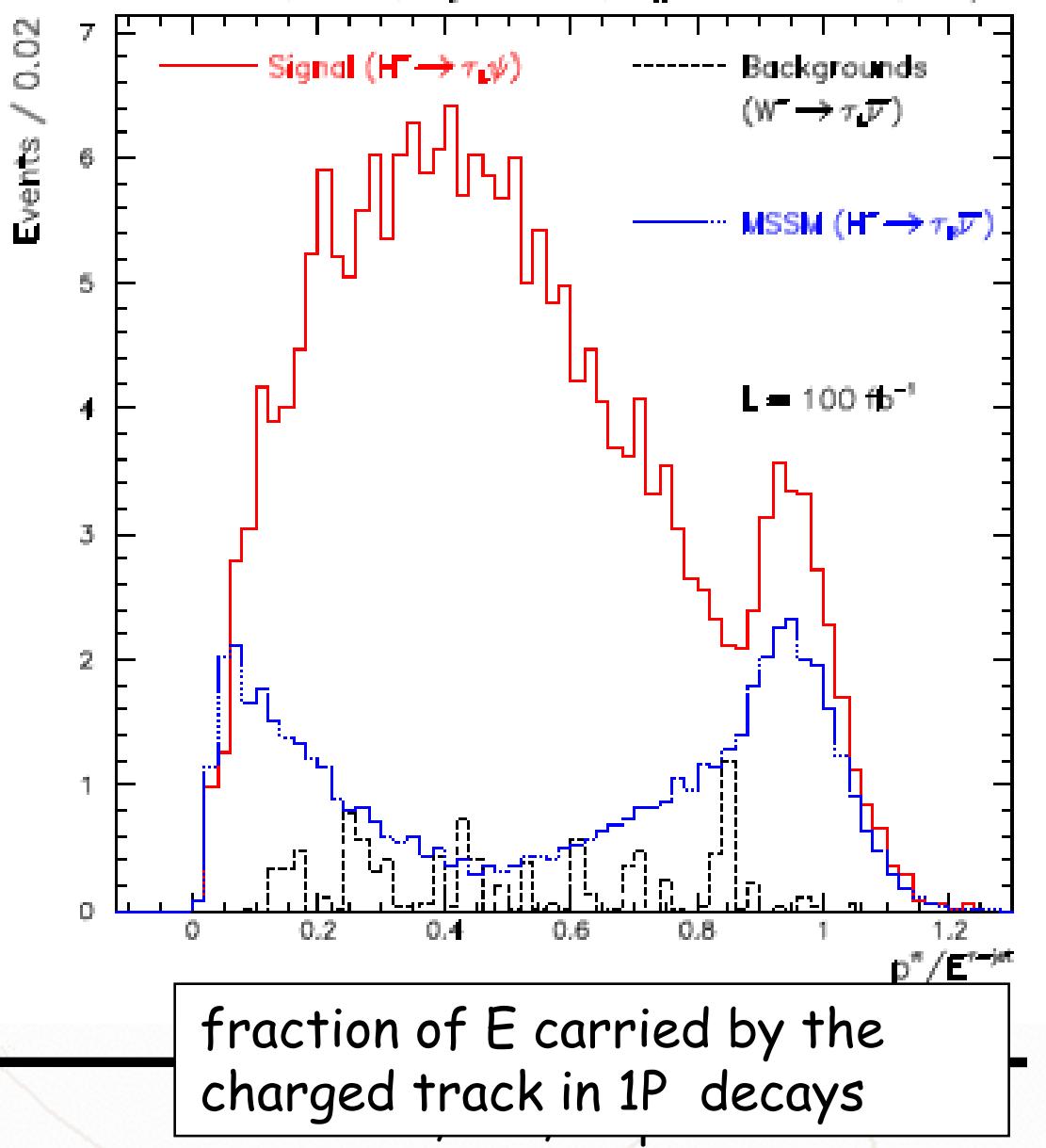




\section{Interesting prospects in SUSY events}

- Tau signatures important in much of the mSUGRA (minimal SuperGravity) parameter space, particularly at high $\tan \beta(>10)$

- in mSUGRA R-parity conserved, all events contain 2 neutralinos escaping the detector $\rightarrow$ one can measure kinematic endpoints in invariant mass distributions rather than mass peaks

- At some points in the parameter space (e.g. funnel) can only observe kinematic endpoints in $T$ invariant mass distributions,

$$
\tilde{\chi}_{2}^{0} \rightarrow \widetilde{\tau}_{1}^{ \pm} \tau^{\mp} \rightarrow \tilde{\chi}_{1}^{0} \tau^{ \pm} \tau^{\mp}
$$

$M_{\max }=f n$ (masses involved SUSY particles)

- Only consider hadronic tau decays. No sharp edge because of $v$, but end-point can still be measured.

- Can use tau polarization measurement to further constrain the underlying SUSY model.

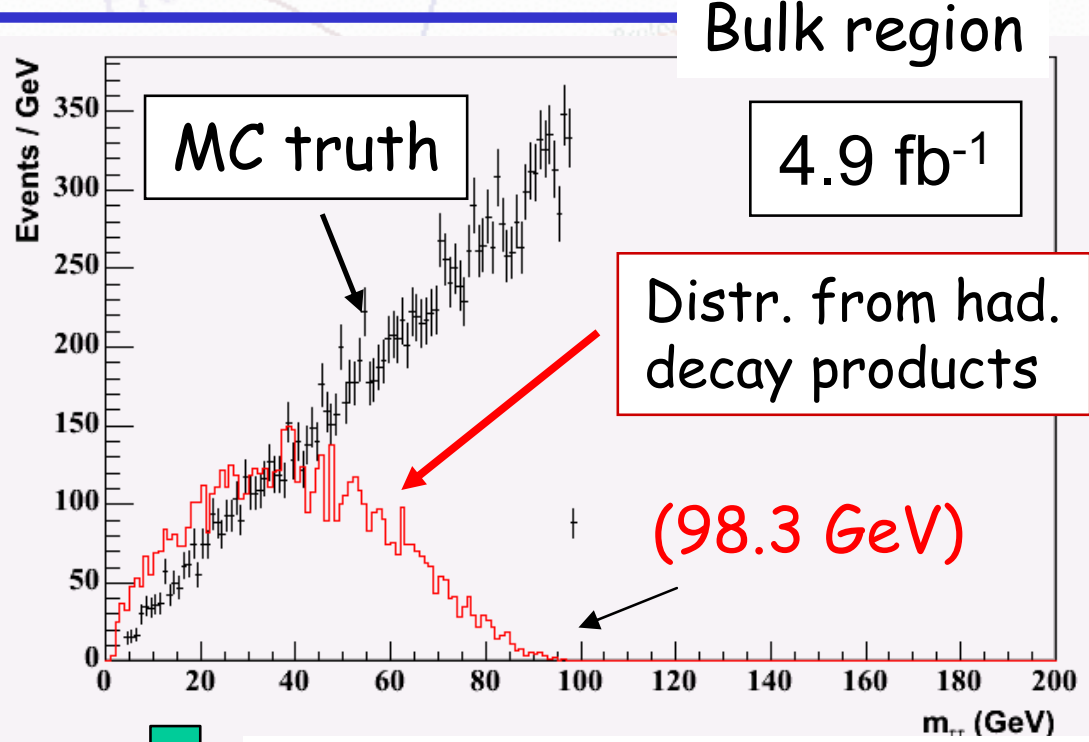

Can fit distorted distribution, and apply this MC fit to the reconstructed distribution

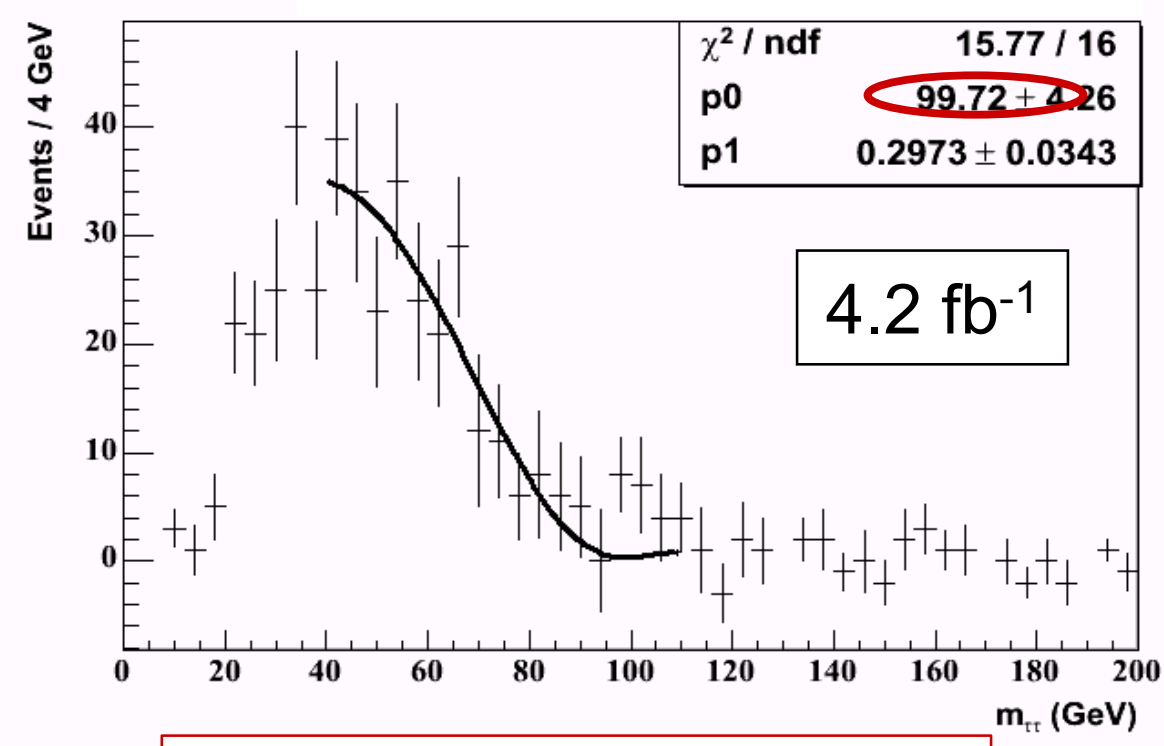

$$
m_{\text {vis }}\left(\tau^{ \pm} \tau^{\mp}\right)-m_{\text {vis }}\left(\tau^{ \pm} \tau^{ \pm}\right)
$$




\section{Conclusions}

- Events with tau's will be observed with the first data of LHC, excellent possibility to understand detector performance.

- Identification of tau leptons will be the key for New Physics discovery:

- SM Higgs in VBF production, $H \rightarrow \tau \tau$

- MSSM Higgs, bbH/A, H/A $\rightarrow \tau \tau$

- MSSM H+ in $+t \rightarrow H^{+} b$ Wb, gb $\rightarrow b H+, H \rightarrow \tau v$

- SUSY signatures with tau's in final states

- extra dimentions(?)... new theories(?)...

- Polarisation measurements should be possible

- Given large dynamic range of required observability in hadronic channel mandatory development of several dedicated algorithms for reconstruction and identification (see Fabien Tarrade talk). 


\section{Backup Slides}




\section{Vector Boson Fusion: $H \rightarrow \tau \tau$}

A number of systematic effects need to be considered.

-uncertainties arise in the simulation of the level of the backgrounds. QCD Zjj events produced with M.E. calculations yield a higher contribution, by at least a factor of two relative to PYTHIA, to the final number of background events.

-uncertainties in detector performance, such as tau-jet and lepton reconstruction efficiencies and rejection factors

-other systematic errors relating to calibration, pileup effects, luminosity measurement,

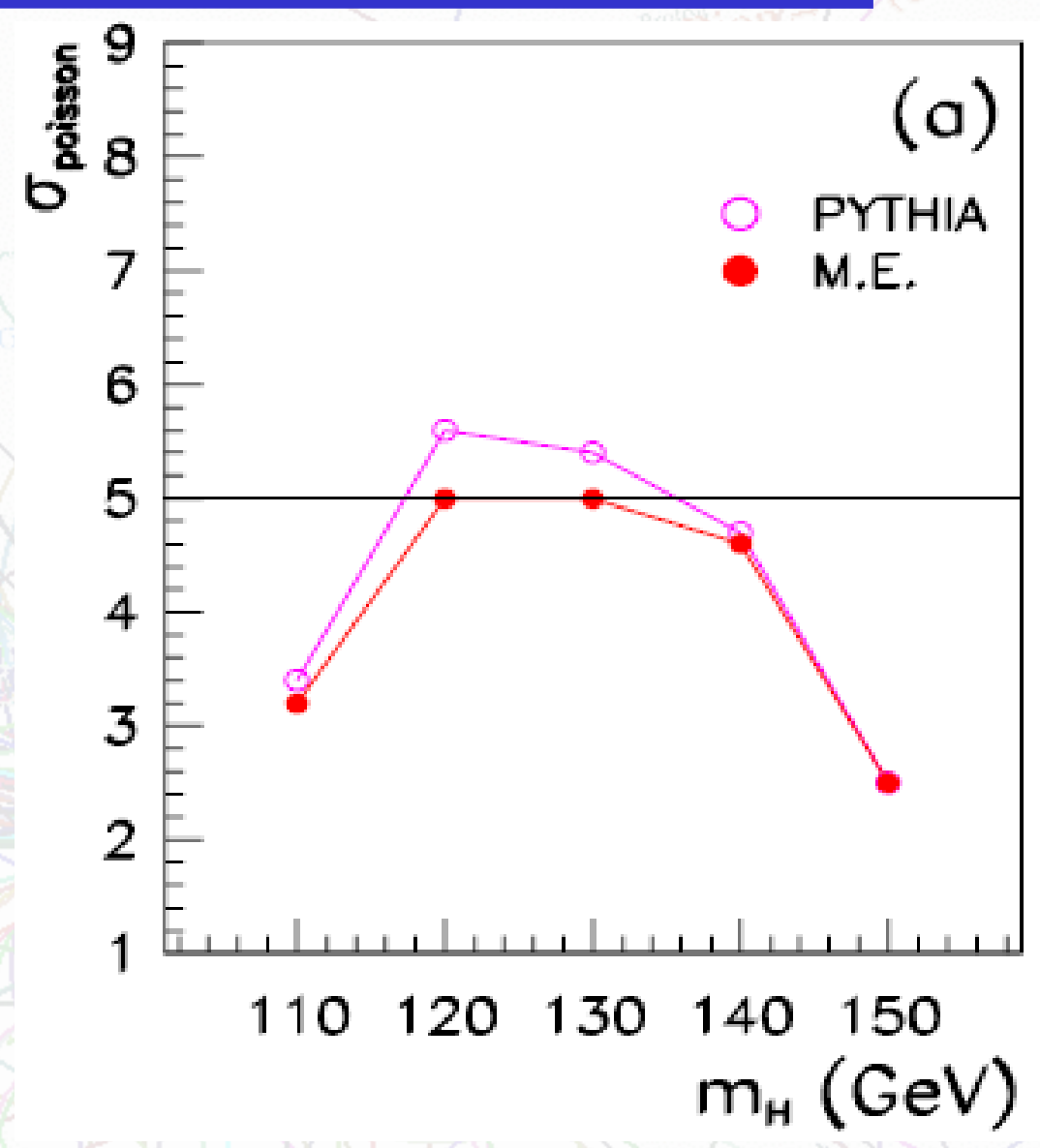

With M.E. QCD Z+jet bkg estimation $30 \mathrm{fb}-1$ 


\section{$H / A \rightarrow \tau \tau$ systematic uncertainties}

- Detector resolution: A worse resolution in the reconstruction of ETmiss results in a broader distribution of the reconstructed mass. In order to estimate this effect, the mass window has been increased by $20 \%$. The signal acceptance has been kept at the value of the standard analysis.

- Identification of the $\tau$ and b-jets: An efficiency of the tau-ID of $55 \%$ has been used. This values has been decrease to $40 \%$, while the jet rejection values remain unchanged. The b-tagging efficiency has been lowered from eff $=0.7$ to 0.6 , while keeping the same rejection factors.

- Jet energy scale: The absolute jet energy scale at the ATLAS are estimated to be known with $3 \%$ accuracy. Therefore, all jet energies in the study have been raised by $3 \%$ to estimate the effect of this uncertainty, which alters the acceptance due to the cuts of the transverse energy of jets used in the event selection.

\begin{tabular}{|l|c|c|c|c|c|}
\hline & $m_{A / H}$ & $\tan \beta$ & Signal & Background & Significance \\
\hline Standard analysis & 600 & 30 & 20.4 & 7.4 & $5.8 \sigma$ \\
& 800 & 45 & 19.6 & 6.8 & $5.8 \sigma$ \\
\hline \hline Detector resolution & 600 & 30 & 20.4 & 9.4 & $5.2 \sigma$ \\
& 800 & 45 & 19.6 & 8.3 & $5.3 \sigma$ \\
\hline$\tau$ identification & 600 & 30 & 14.9 & 7.5 & $4.3 \sigma$ \\
and b-tagging & 800 & 45 & 13.8 & 5.7 & $4.4 \sigma$ \\
\hline jet energy scale & 600 & 30 & 18.6 & 8.6 & $5.0 \sigma$ \\
& 800 & 45 & 16.7 & 7.3 & $4.8 \sigma$ \\
\hline
\end{tabular}

Table 10: Study of the influence of systematic uncertainties of the significance of the channel $(\mathrm{b} \overline{\mathrm{b}}) \mathrm{A} / \mathrm{H} \rightarrow(\mathrm{b} \overline{\mathrm{b}}) \tau($ had $) \tau($ had $)$. 


\section{Closer look at charged $\mathrm{H}^{ \pm} \rightarrow \tau \nu$}

Table 1: The statistical precision on the mass determination in the $H^{ \pm} \rightarrow \tau \nu$ channel. The reference masses are listed in the first column. The reconstructed masses $<m>(\mathrm{GeV})$ and the corresponding precision $\delta m(\mathrm{GeV})$ are calculated for 100 and $300 \mathrm{fb}^{-1}$. We take $\tan \beta=45$. The statistical precision deteriorates as the Higgs mass increases because of the reduction in rate.

\begin{tabular}{ccccc}
\hline$m_{H^{ \pm}}(\mathrm{GeV})$ & \multicolumn{2}{l}{$\mathcal{L}=100 \mathrm{fb}^{-1}$} & \multicolumn{2}{c}{$\mathcal{L}=300 \mathrm{fb}^{-1}$} \\
\hline$m_{0}$ & $<m>$ & $\delta m$ & $\langle m>$ & $\delta m$ \\
225.9 & 226.4 & 3.0 & 226.4 & 1.7 \\
271.1 & 271.0 & 3.4 & 271.1 & 2.0 \\
317.8 & 318.3 & 5.2 & 318.3 & 3.0 \\
365.4 & 365.5 & 7.8 & 365.7 & 4.6 \\
413.5 & 413.6 & 7.7 & 413.8 & 4.5 \\
462.1 & 462.3 & 10.2 & 462.6 & 6.0 \\
510.9 & 511.5 & 13.0 & 511.9 & 7.4 \\
\hline \hline
\end{tabular}

Table 2: The systematic effects on the mass determination in the $H^{ \pm} \rightarrow \tau \nu$ channel are small. Columns 2 and 3 show the statistical uncertainties for an integrated luminosity of $300 \mathrm{fb}^{-1}$. Columns 4 and 5 include the systematic uncertainties. The total

Systematic uncertainties: -the shape of the background -the background rate (known to $5 \%$ )

-the energy scale $-1 \%$ for jets and $0.1 \%$ for photons, electrons and muons.

Anna Kaczmarska uncertainties are dominated by the statistical errors.

\begin{tabular}{ccccc}
\hline \hline$m_{H^{ \pm}}(\mathrm{GeV})$ & \multicolumn{2}{l}{ No systematics } & \multicolumn{2}{c}{ With systematics } \\
\hline & $<m>$ & $\delta m$ & $\langle m\rangle$ & $\delta m$ \\
225.9 & 226.4 & 1.7 & 225.9 & 1.7 \\
271.1 & 271.1 & 2.0 & 270.9 & 2.3 \\
317.8 & 318.3 & 3.0 & 319.9 & 3.5 \\
365.4 & 365.7 & 4.6 & 365.2 & 4.7 \\
413.5 & 413.8 & 4.5 & 414.9 & 4.7 \\
462.1 & 462.6 & 6.0 & 460.8 & 6.3 \\
510.9 & 511.9 & 7.4 & 511.7 & 9.2 \\
\hline \hline
\end{tabular}




\section{Closer look at charged $H^{ \pm} \rightarrow \tau v$}

Table 4: The overall precisions on the rate determination in the $H^{ \pm} \rightarrow \tau \nu$ channel for $\mathcal{L}=30,100$ and $300 \mathrm{fb}^{-1}$. The total number of background events is $B=6.7$ for $30 \mathrm{fb}^{-1}[10]$. The number of signal events listed in the second column correspond to an integrated luminosity of $30 \mathrm{fb}^{-1}[10]$.

\begin{tabular}{ccccc}
\hline \hline$\left(m_{H^{ \pm}}[\mathrm{GeV}], \tan \beta\right)$ & $S \equiv$ Signal events & \multicolumn{3}{c}{$\Delta(\sigma \times B R) /(\sigma \times B R)(\%)$} \\
\hline & $30 \mathrm{fb}^{-1}$ & $30 \mathrm{fb}^{-1}$ & $100 \mathrm{fb}^{-1}$ & $300 \mathrm{fb}^{-1}$ \\
200,30 & 46.3 & 18.6 & 14.2 & 11.6 \\
250,40 & 60.3 & 16.9 & 13.3 & 11.2 \\
300,45 & 70.5 & 16.0 & 12.8 & 11.0 \\
350,25 & 18.8 & 28.7 & 19.9 & 14.1 \\
400,35 & 30.6 & 22.3 & 16.2 & 12.4 \\
450,60 & 66.9 & 16.3 & 12.9 & 11.1 \\
500,50 & 36.2 & 20.7 & 15.3 & 12.0 \\
\hline \hline
\end{tabular}

The main systematic error would come from the knowledge of the luminosity $(+-10 \%$.) 

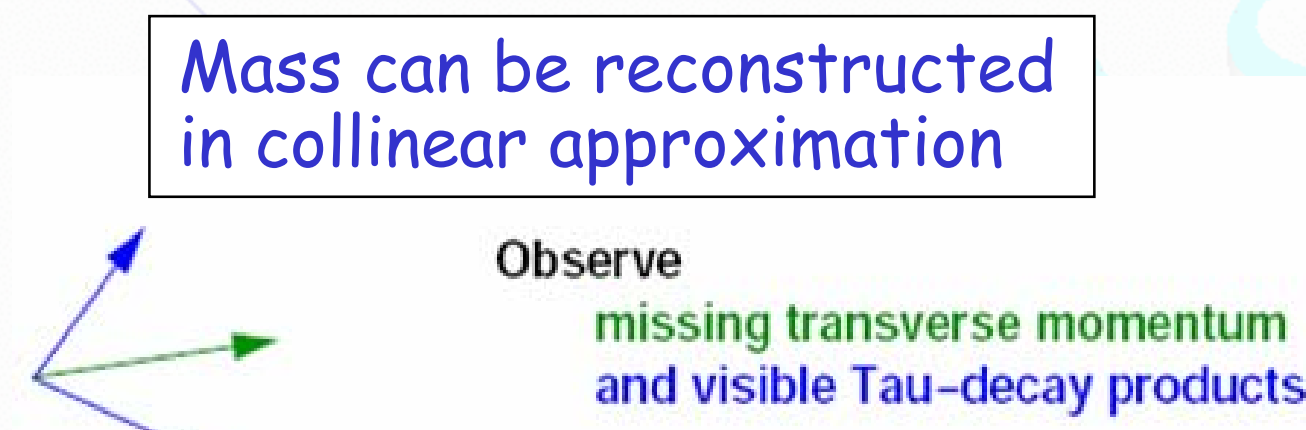

Assume Tau decay products collinear with original Tau

Solve 2 linear equations for the neutrinos

Taus can be reconstructed

Higgs can be reconstructed

$$
\begin{aligned}
& x_{\tau h}=\frac{h_{x} l_{y}-h_{y} l_{x}}{h_{x} l_{y}+\not p_{x} l_{y}-h_{y} l_{x}-\not p_{y} l_{x}} \\
& x_{\tau l}=\frac{h_{x} l_{y}-h_{y} l_{x}}{h_{x} l_{y}-\not p_{x} h_{y}-h_{y} l_{x}+p_{y} h_{x}}
\end{aligned}
$$

$\mathrm{x}_{\tau}=$ momentum fraction carried by tau decay products
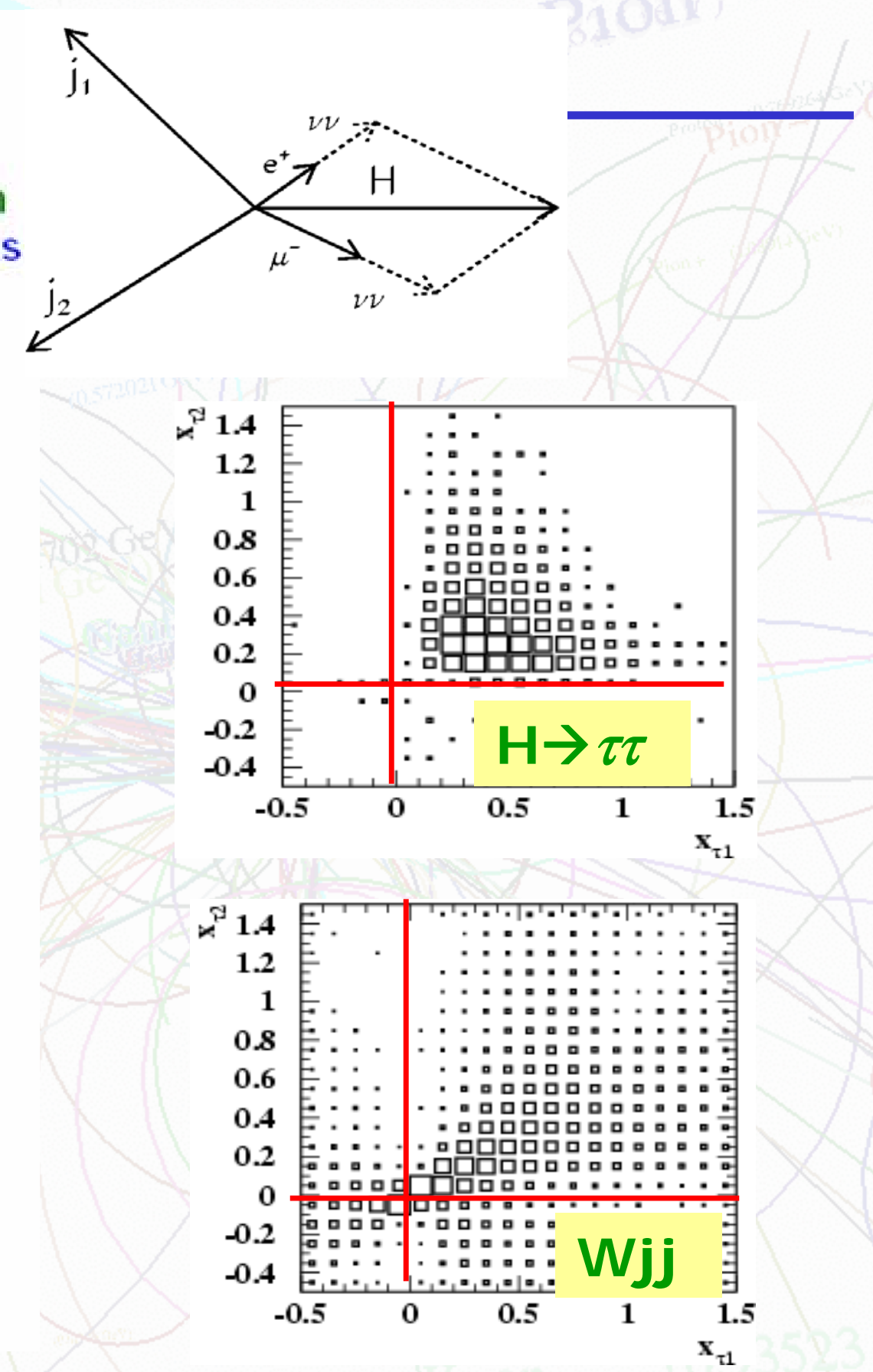

Tau 06, Pisa, September 2006 


$$
m_{T}=\sqrt{2 p_{T}^{\tau} p_{T}[1-\cos (\Delta \phi)]}
$$

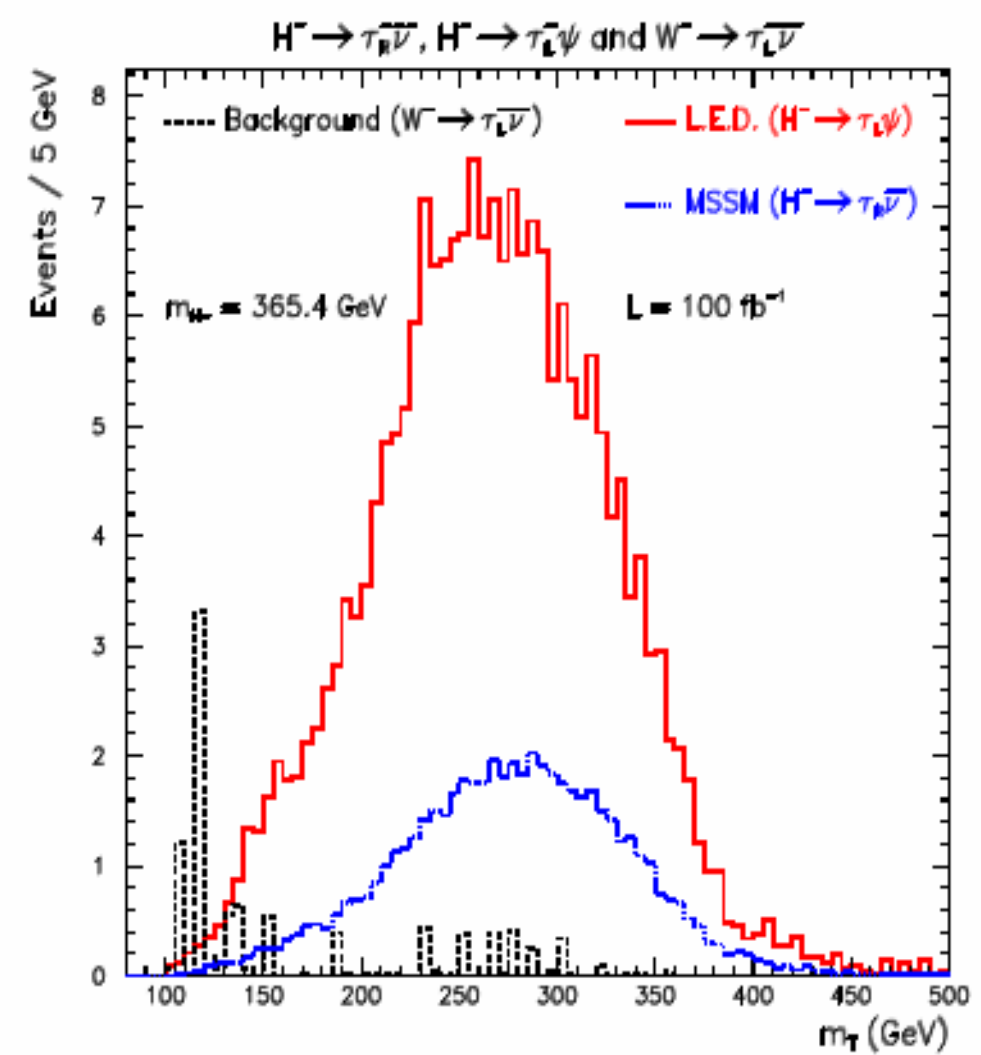

$\Rightarrow$ Observation of signal in $m_{T}$ distribution is not sufficient to distinguish between 2HDM (MSSM) and L.E.D.
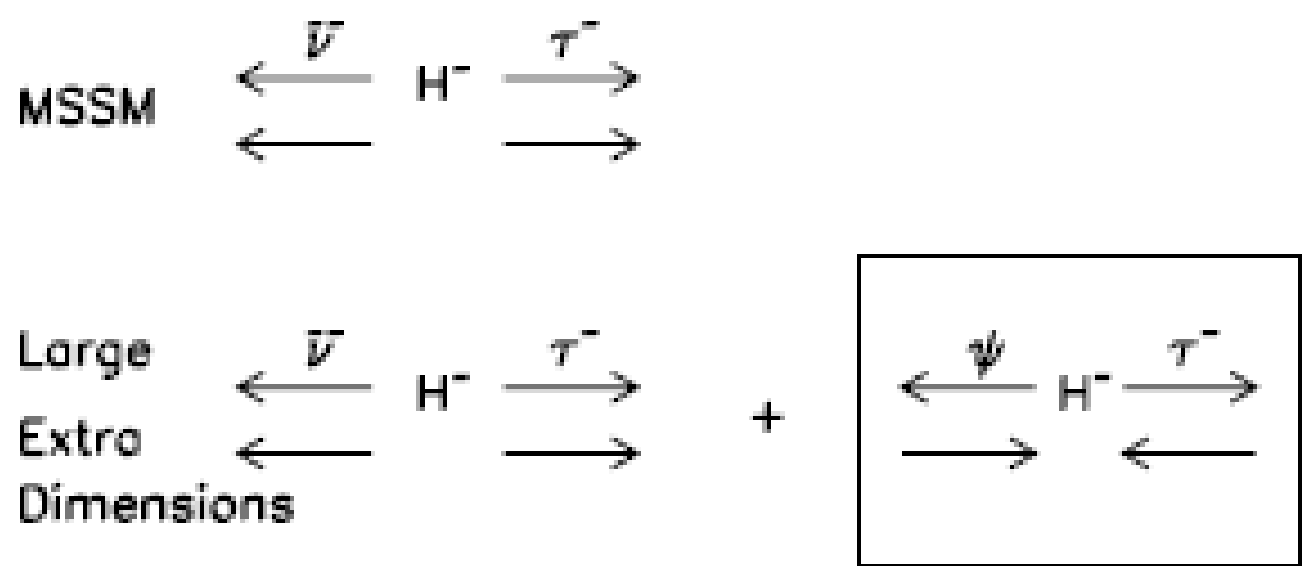

\section{Backgrounds}

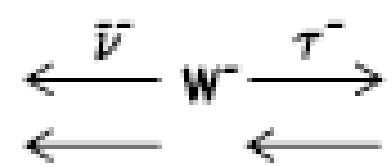

\section{One-prong $\tau^{-}$decoys to $\pi^{-} \nu$}

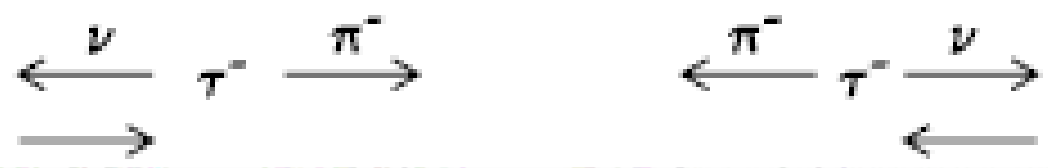

FIG. 6: Polarization of the decay $\tau$ from $H^{ \pm}$in MSSM and in models with a singlet neutrino in large extra dimensions. In the latter case, both left and right handed $\tau$ 's can be produced with some polarization asymmetry. In the backgrounds, the $\tau$ comes from the decay of the $W^{ \pm}$. The signal to be studied is in the box - the polarization of the decay $\tau$ in this signal is the same as in the background. Thus, $\tau$ polarization effects would not help in suppressing the backgrounds but they may help distinguish between the 2HDM and other models. 


\section{Last but not least: life-time measurement}

The tau lepton lifetime:

$$
\begin{aligned}
& 289.4 \pm 0.91 \text { (stat) } \pm 0.90 \text { (syst) fs } \\
& \text { (preliminary) BABAR, SLAC-PUB-11317 } \\
& 290.9 \pm 1.4 \text { (stat) } \pm 1.0 \text { (syst) fs } \\
& \text { DELPHI, Nucl. Phys. Proc. Suppl. } 144 \text { (2005) } 105
\end{aligned}
$$

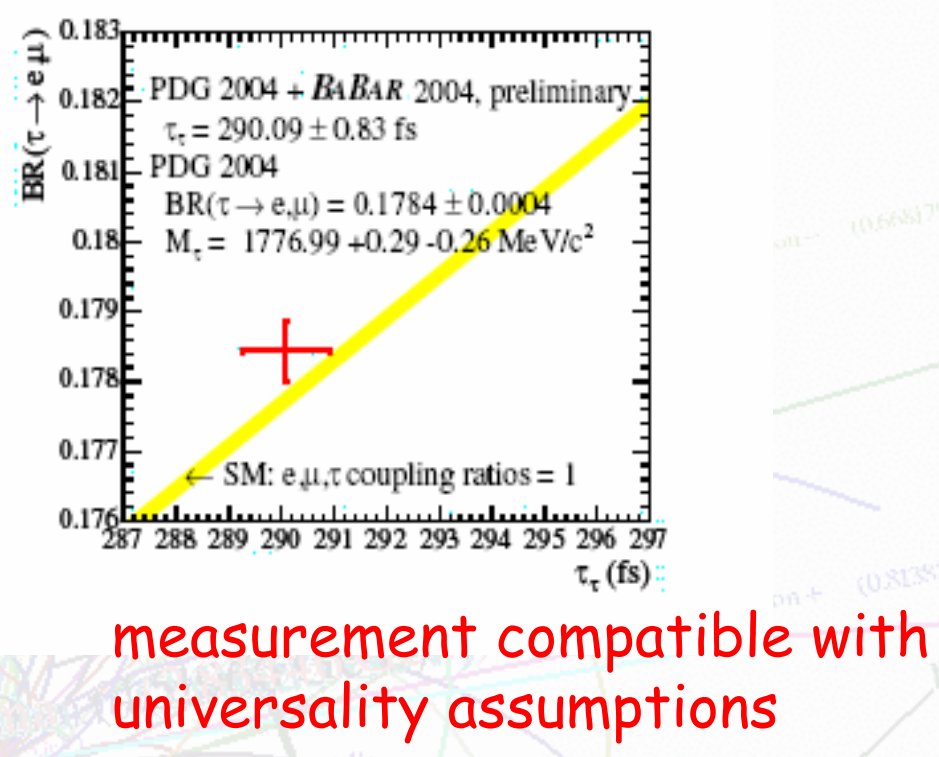

With ATLAS, use $Z \rightarrow \tau \tau$ or $W \rightarrow \tau v$ events, three-prong decays,

$Z \rightarrow \tau \tau$ events: $\rightarrow$ statistical error of $1.8 \mathrm{fs}$,

D. Cavalli \& B. Osculati, ATL-PHYS-2000-014 not competitive with other measurements

$W \rightarrow \tau v$ events: $\rightarrow$ statistical error of $0.25-0.35 \mathrm{fs}$, competitive with measurements at $e^{+} e^{-}$colliders $\rightarrow$ expect 780000 hadronic tau-decay events for $30 \mathrm{fb}^{-1}$ $\rightarrow$ not proven yet that systematic error can be kept under control 


\section{$\tilde{\tau}_{1}^{ \pm}$NLSP in large part of MSSM parameter space}

\section{Decay chains often end with:}

$$
\tilde{\tau}_{1}^{ \pm} \rightarrow \tau^{ \pm} \tilde{Z}_{1} \quad \begin{aligned}
& \text { : Hadronic decays: sensitive to tau polarization } \\
& \pi^{ \pm} \nu(12.5 \%), \rho^{ \pm} \nu(26 \%), a_{1}^{ \pm} \nu(7.5 \%)
\end{aligned}
$$

Tau polarization depends on composition of LSP

\section{LSP composition dependence of tau polarization:}

$$
\begin{aligned}
& \text { Polarization } P_{\tau}=\frac{\left(a_{11}^{R}\right)^{2}-\left(a_{11}^{L}\right)^{2}}{\left(a_{11}^{R}\right)^{2}+\left(a_{11}^{L}\right)^{2}} \text {, } \\
& \text { with } a_{11}^{R}=-\frac{2 g}{\sqrt{2}} N_{11} \tan \theta_{W} \sin \theta_{\tau}-\frac{g m_{\tau}}{\sqrt{2} m_{W} \cos \beta} N_{13} \cos \theta_{\tau} \text {. } \\
& a_{11}^{L}=\frac{g}{\sqrt{2}}\left[N_{12}+N_{11} \tan \theta_{W}\right] \cos \theta_{\tau}-\frac{g m_{\tau}}{\sqrt{2} m_{W} \cos \beta} N_{13} \sin \theta_{\tau},
\end{aligned}
$$

LSP composition $\tilde{Z}_{1}=N_{11} \tilde{B}+N_{12} \tilde{W}+N_{13} \tilde{H}_{1}+N_{14} \tilde{H}_{2}$.

$$
\tilde{\tau}_{1}^{ \pm} \text {composition } \tilde{\tau}_{1}=\tilde{\tau}_{R} \sin \theta_{\tau}+\tau_{L} \cos \theta_{\tau}
$$

- Universal SUGRA models:

$$
P_{\tau} \simeq+1
$$

- For most non-universal SUGRA models:

$$
P_{\tau} \simeq \cos ^{2} \theta_{\tau}-\sin ^{2} \theta_{\tau}
$$

- AMSB models:

$$
P_{\tau} \simeq-1
$$

- For many GMSB models ( $\tilde{\tau}_{1} \rightarrow \tau \tilde{G}$ decay):

$$
P_{\tau}=\sin ^{2} \theta_{\tau}-\cos ^{2} \theta_{\tau}
$$




\section{Sugra models}

$\rightarrow$ Reminder:

- Minimal Sugra (mSUGRA) has 4 parameters + a sign:

- $m_{0}$ : common scalar mass at GUT scale

- $m_{1 / 2}:$ common gaugino mass at GUT scale

- A: common trilinear Higgs sfermion-sfermion coupling at GUT scale

- tg $\beta$ : ratio of the Higgs vacuum expectation values

- $\operatorname{sgn}(\mu): \mu$ being the SUSY conserving Higgsino mass 


\section{mSUGRA: selected points}

- DC1 bulk region point (new underlying event in generation)

- $\mathrm{m}_{0}=100 \mathrm{GeV}, \mathrm{m}_{1 / 2}=300 \mathrm{GeV}, \mathrm{A}_{0}=-300 \mathrm{GeV}, \tan \beta=6, \operatorname{sgn}(\mu)=+$

- LSP is mostly bino, light $I_{R}$ enhance annihilation. 'Bread and butter' region for the LHC experiments

- Ilq distributions, tau-tau measurements, third generation squarks (both tau

identification and $\mathrm{B}$ tagging improved)

- Coannihilation point

- $\mathrm{m}_{0}=70 \mathrm{GeV}, \mathrm{m}_{1 / 2}=350 \mathrm{GeV}, \mathrm{A}_{0}=0 \mathrm{GeV}, \tan \beta=10, \operatorname{sgn}(\mu)=+$

- LSP is pure bino. LSP/sparticle coannihilation. Small slepton-LSP mass difference gives soft leptons in the final state

- Focus point

- $\mathrm{m}_{0}=3350 \mathrm{GeV}, \mathrm{m}_{1 / 2}=300 \mathrm{GeV}, \mathrm{A}_{0}=0 \mathrm{GeV}, \tan \beta=10, \operatorname{sgn}(\mu)=+$

- LSP is Higgsino, near $\mu^{2}=0$ bound. Heavy sfermions; all squarks and sleptons have mass $>2 \mathrm{TeV}$, negligible $\mathrm{FCNC}, \mathrm{CP}, \mathrm{g}_{\mu}-2$, etc. Complex events with lots of heavy flavor

- Funnel region point

- $\mathrm{m}_{0}=320 \mathrm{GeV}, \mathrm{m}_{1 / 2}=375 \mathrm{GeV}, \mathrm{A}_{0}=0 \mathrm{GeV}, \tan \beta=50, \operatorname{sgn}(\mu)=+$

- wide $\mathrm{H}, \mathrm{A}$ for $\tan \beta \gg 1$ enhance annihilation. Heavy Higgs resonance (funnel); main annihilation chain into bb pairs

- dominant tau decays

- Low mass point at limit of Tevatron Runll reach

- $\mathrm{m}_{0}=200 \mathrm{GeV}, \mathrm{m}_{1 / 2}=160 \mathrm{GeV}, \mathrm{A}_{0}=-400 \mathrm{GeV}, \tan \beta=10, \operatorname{sgn}(\mu)=+$

- big cross section, but events rather similar to top

- measure SM processes in presence of SUSY background to show detector is understood 


\section{Inclusive analysis}

Select events with at least 4 jets and Missing $E_{T}$ A simple variable

$M_{\text {eff }}=P_{t, 1}+P_{t, 2}+P_{t, 3}+P_{t, 4}+E_{T}$

At high $M_{\text {eff }}$ non-SM signal rises above background note scale

Peak in $M_{\text {eff }}$ distribution correlates well with SUSY mass scale

$M_{\mathrm{SUSY}}=\min \left(M_{\bar{u}}, M_{\bar{g}}\right)$

Will determine gluino/squark masses to $\sim 15 \%$

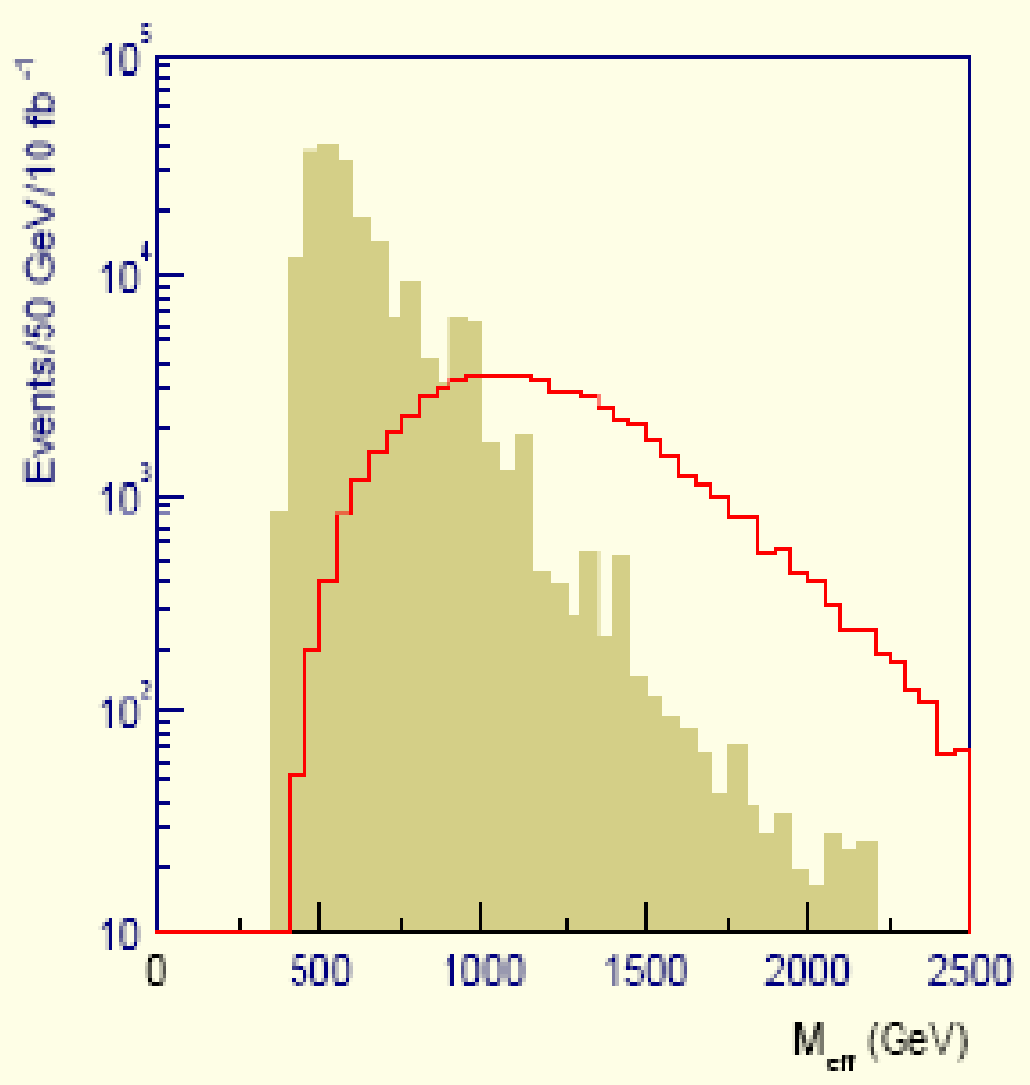




\section{Tau decay modes}

$\Rightarrow \mathrm{c} \tau \sim=87 \mu \mathrm{m}, \mathrm{m}_{\tau}=1.78 \mathrm{GeV} / \mathrm{c}^{2}$

- Leptonical decays

Quite often taus are produced in pairs: $42 \%$ of final states contains two "tau-jet"

- $\tau->\mathrm{e}(\mu) \vee v: \sim 35.2 \%$

- Identification done through the final lepton

- Hadronical decays

-1 prong

$$
\tau \rightarrow v_{\tau}+\pi^{+/-}+n\left(\pi^{0}\right): 49.5 \%
$$

3 prongs

$$
\tau \rightarrow v_{\tau}+3 \pi^{+/-}+n\left(\pi^{0}\right): 15.2 \%
$$

- ${ }^{6} \tau$-jet" is produced

\begin{tabular}{c|c}
\hline \hline$\tau \tau$ decay mode & BR \\
\hline$\ell \ell \nu$ & $12 \%$ \\
$\ell$ jet $\nu$ & $46 \%$ \\
jet jet $\nu$ & $42 \%$ \\
\hline \hline
\end{tabular}

-tau jets at LHC:

-very collimated

$.90 \%$ of the energy is contained in a 'cone' of radius $\mathrm{R}=0.2$ around the jet direction for ET>50 GeV

- Low multiplicity

-One, three prongs

-Hadronic, EM energy deposition

- Charged pions

-Photons from $\pi^{0}$ 


\section{The ATLAS detector at LHC}

$\gamma$-electrons, jets, $E_{t}^{\text {miss }}, \sigma_{E}$
$\eta \mid$ coverage, b-tagging

Thin Superconducting Solenoid $(B=2 T)$

LAr EM Calorimeter: $L \times R=13.3 \mathrm{~m} \times 2.25 \mathrm{~m}$ $|\eta| \leq 3.2$ (4.9) $\sigma_{E} / E=10 \% / \sqrt{ } E \oplus 0.7 \%$ PB-LAr

Hadronic Calorimeter : Endcaps LArg Barrel Scintillator-tile $L \times R=12.2 \mathrm{~m} \times 4.25 \mathrm{~m}$ $\sigma_{E} / E=50 \% / \sqrt{ } E \oplus 3 \%(|\eta| \leq 3)$

Large Superconducting Air-Core Toroids

Muon Spectrometer $L \times R=25(46) m \times 11 m$ $\sigma($ EtMiss $)=0.46 * \operatorname{sqrt}($ SumET $))$
Inner Detector :

Semiconductor Pixel and Strips Straw Tube Tracking Detector (TRT) $L \times R=7 m \times 1.15 m$ $\sigma_{R \phi}=12-16 \mu \mathrm{m}, \sigma_{\mathrm{Z}}=66-580 \mu \mathrm{m}$

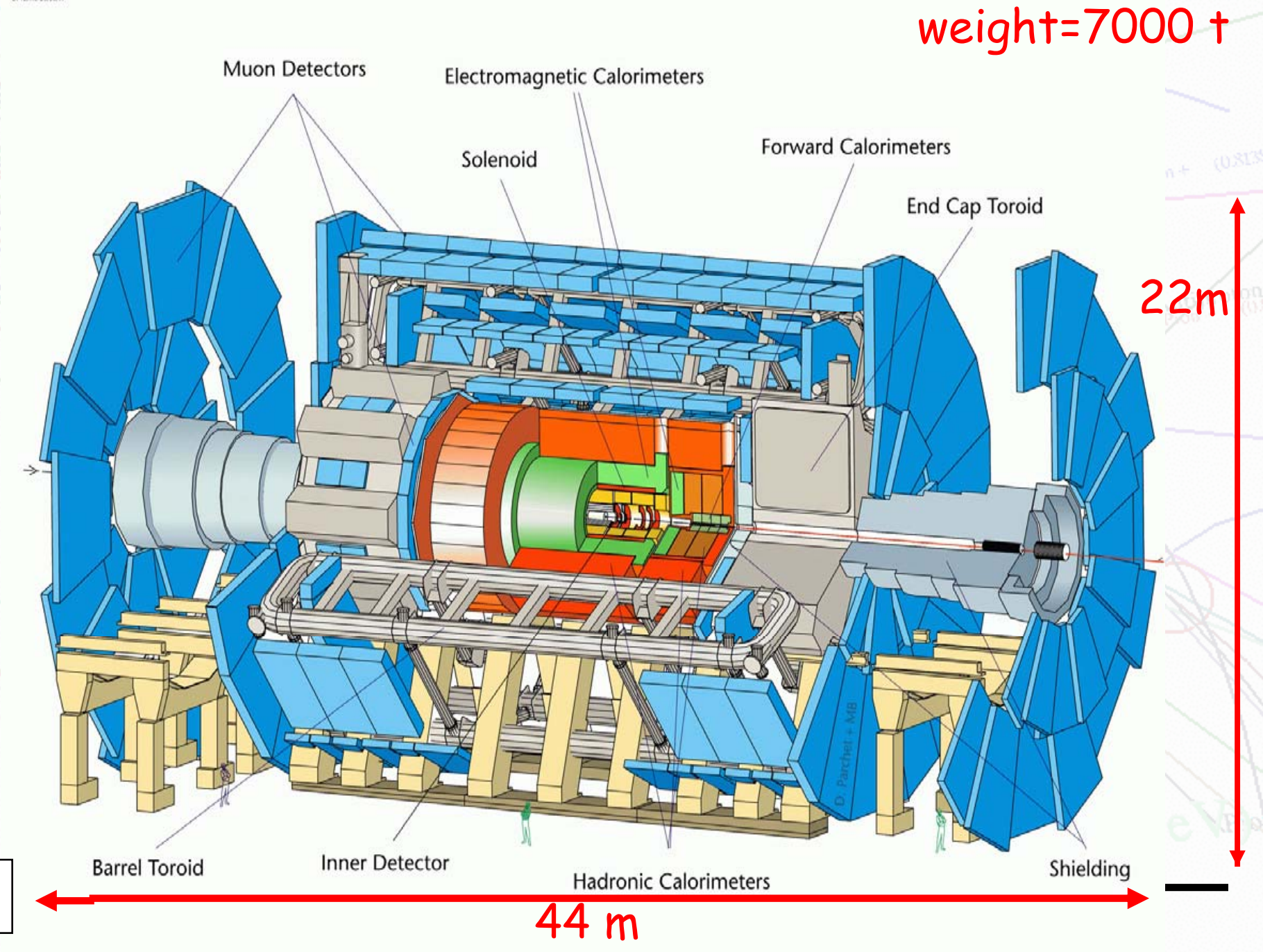




\section{SM Higgs with mass below $200 \mathrm{GeV}$}

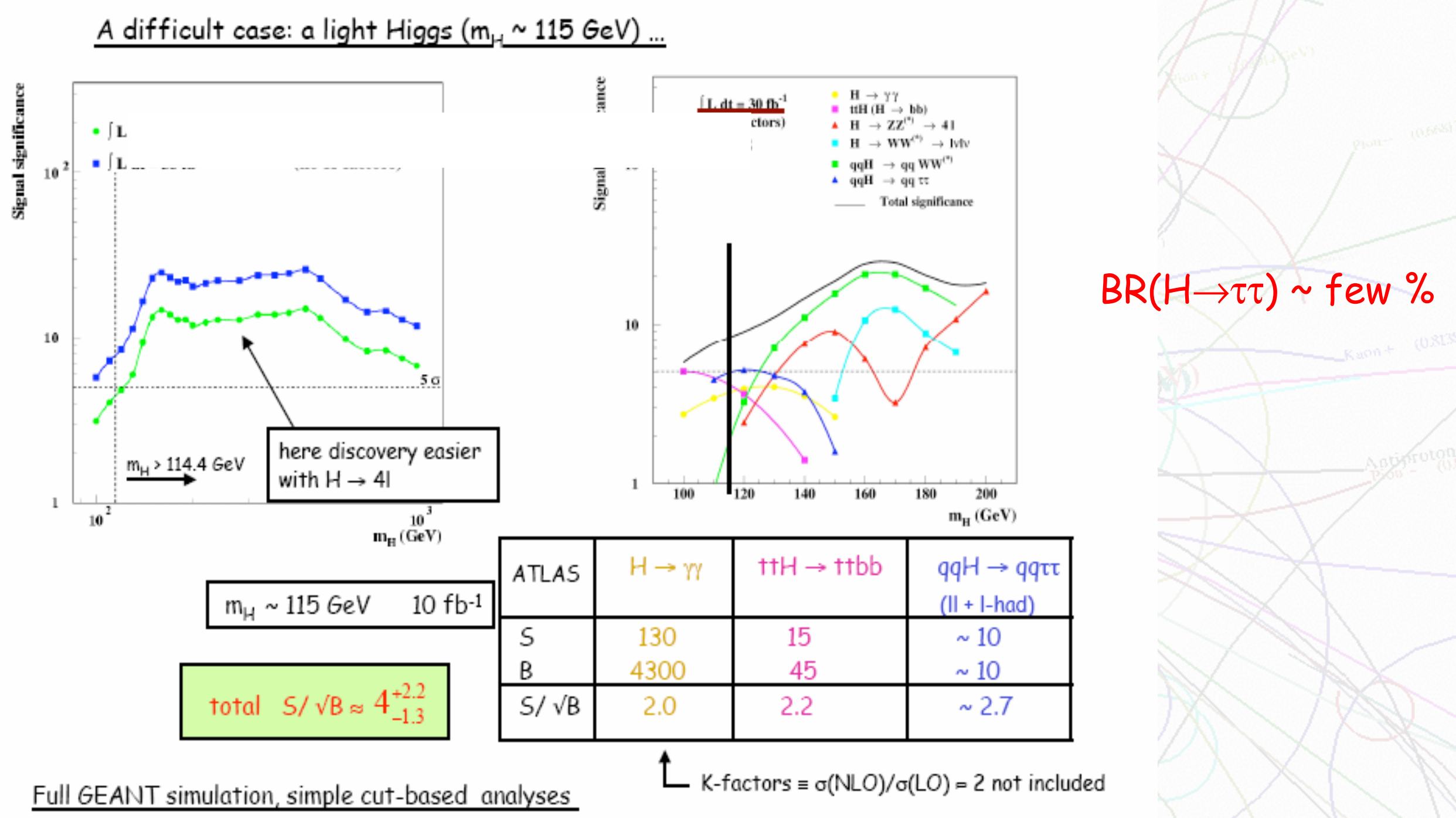

If clean had-had channel observable, sensitivity to Higgs parity might be established but which production process use to allow to trigger such events? 


\section{SM Higgs production at LHC}

\section{gg fusion}

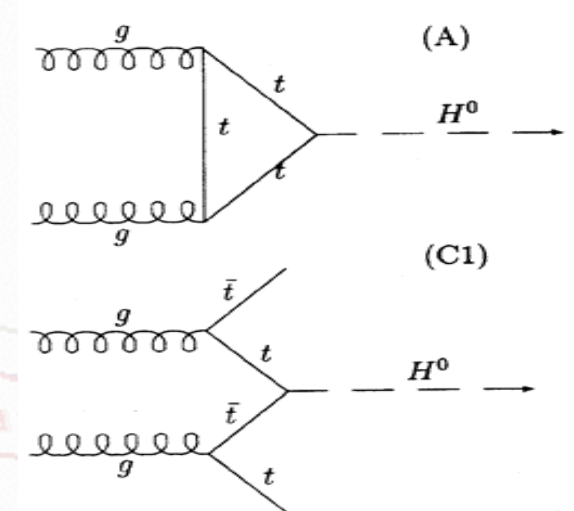

associated $\mathrm{ttH}$
WW/ZZ fusion

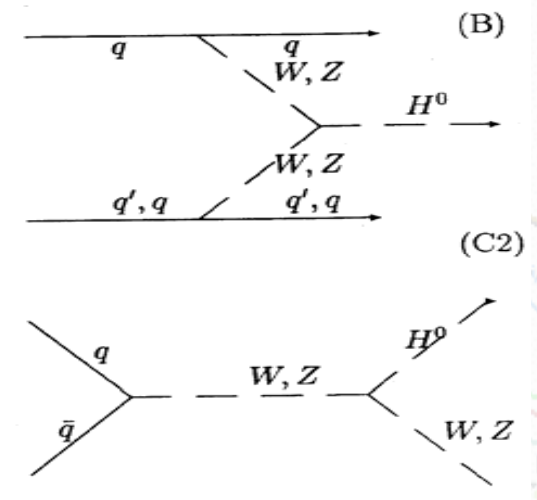

associated $\mathrm{WH}, \mathrm{ZH}$

$\Rightarrow m_{H}<2 m_{Z}:$

- $\mathrm{H} \rightarrow \mathrm{r}$

- $\mathrm{HtH} \rightarrow \ell \vee \mathrm{bb}+\mathrm{X}$

- $\mathrm{H} \rightarrow \mathrm{ZZ} \mathrm{Z}^{*} \rightarrow 4 \ell$

- $\mathrm{H} \rightarrow \mathrm{WW}^{*} \rightarrow \ell v \ell v$

- $W H \rightarrow W W W^{\star} \rightarrow \ell v \ell v \ell v, \ell^{ \pm} \ell^{ \pm} v v j j$

$\Rightarrow m_{H}>2 m_{Z}:$

- Main channel is $H \rightarrow Z Z \rightarrow 4 \ell$ (gold-plated)

- $\mathrm{H} \rightarrow \mathrm{ZZ} \rightarrow \ell \ell \mathrm{vv}$

- $H \rightarrow Z Z \rightarrow \ell \ell j j$

- $H \rightarrow W W \rightarrow \ell v j j$ $m_{H}>300 \mathrm{GeV}$ forward jet tag
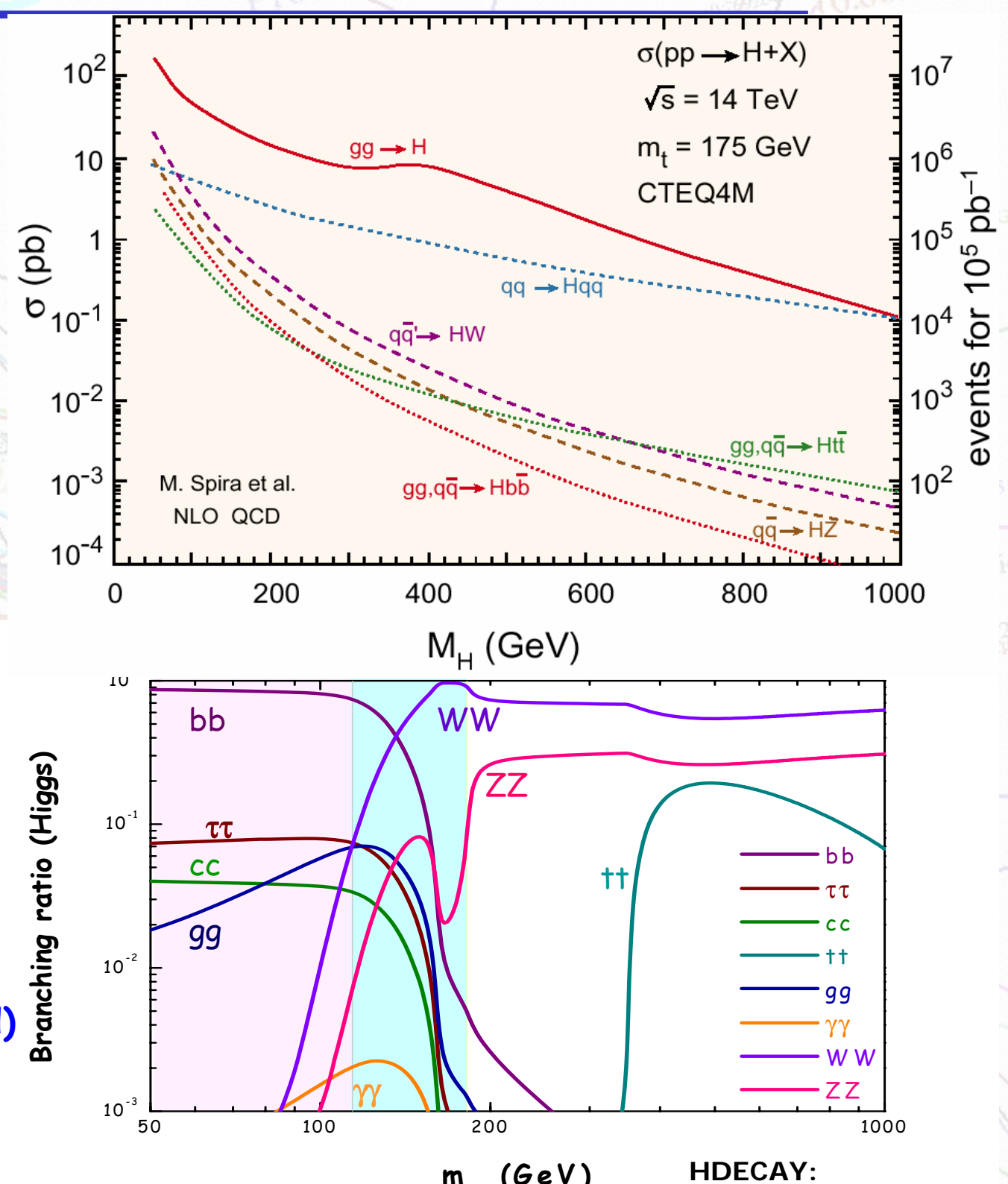

fully hadronic final states dominate but Anna Kaczmarska cannot be extracted from large QCD backgrounds 


\section{MSSM Higgs searches}

\section{Two Higgs Doublets with 5 physical states}

- 2 CP-even neutral Higgs bosons $\mathrm{h}^{0}, \mathrm{H}^{0}$

- 1 CP-odd neutral Higgs boson $\quad \mathrm{A}^{0}$

- 2 charged Higgs bosons

$\mathrm{H}^{ \pm}$

- free parameters $\tan \beta=v_{1} / v_{2}$

$\mathrm{m}_{\mathrm{A}}$ tree level:

$$
\begin{aligned}
& \mathrm{m}_{\mathrm{h}} \leq \mathrm{m}_{\mathrm{Z}} \\
& \mathrm{m}_{\mathrm{A}} \leq \mathrm{m}_{\mathrm{H}} \\
& \mathrm{m}_{\mathrm{W}} \leq \mathrm{m}_{\mathrm{H} \pm}
\end{aligned}
$$

rad. corrected: $m_{h}<130 \mathrm{GeV}$

many possible search channels@LHC

$\mathrm{h} \rightarrow \gamma \gamma, \quad$ tt $\mathrm{h} \rightarrow \mathrm{tt} b \mathrm{~b}, \mathrm{H} \rightarrow \mathrm{ZZ}^{(*)} \rightarrow 4 \ell$ like SM

$\mathrm{A} / \mathrm{H} \rightarrow \mu \mu, \tau \tau, \mathrm{tt}, \quad \mathrm{H}^{ \pm} \rightarrow \tau \mathrm{v}, \mathrm{cs}, \mathrm{tb}$ $\mathrm{H} \rightarrow \mathrm{hh}, \mathrm{A} \rightarrow \mathrm{Zh}$ typical for

MSSM
$\mathrm{A} / \mathrm{H} \rightarrow \chi_{2}^{0} \chi_{2}^{0}$

$\chi_{2}^{0} \rightarrow \mathrm{h} \chi^{0}{ }_{1}$

if SUSY particles

accessible 


\section{What data samples in 2007 ?}

\section{ATLAS preliminary $\sqrt{ } s=900 \mathrm{GeV}, \mathrm{L}=10^{29} \mathrm{~cm}^{-2} \mathrm{~s}^{-1}$ Trigger and analysis efficiencies included}

$30 \%$ data taking efficiency included (machine plus detector)

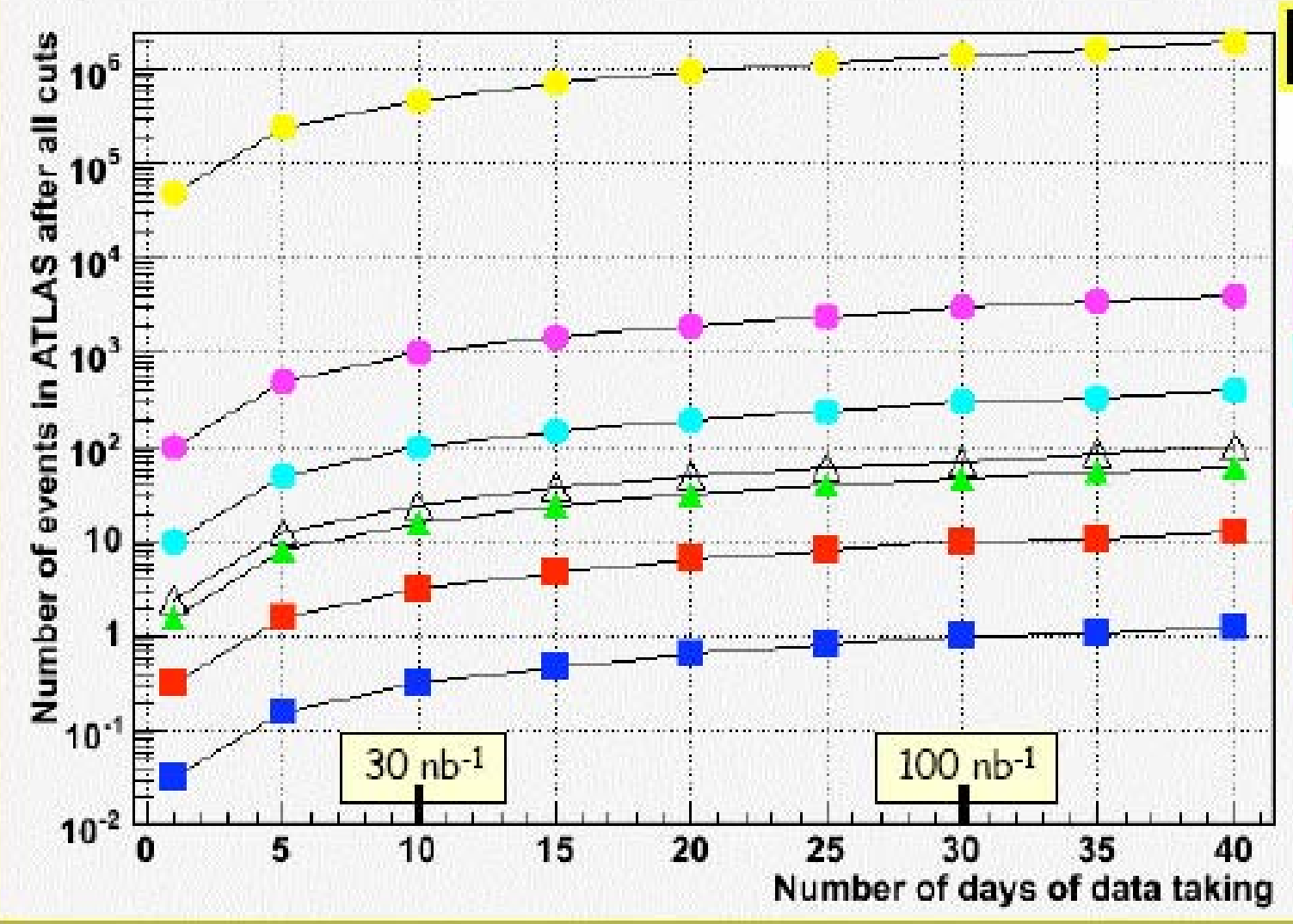

\section{Jets $P_{T}>15 \mathrm{GeV}$}

(b-jets: $1.5 \%)$

\section{Jets $\mathrm{PT}_{\mathrm{T}}>50 \mathrm{GeV}$}

Jets $\mathrm{PT}_{\mathrm{T}}>70 \mathrm{GeV}$

$\mathbf{Y} \rightarrow \mu \mu \quad \mathrm{J} / \psi \rightarrow \mu \mu$

\section{$W \rightarrow e v, \mu v$}

$Z \rightarrow e e, \mu \mu$

+1 million minimum-bias/day

Start to commission triggers and detectors with collision data (minimum bias, jets, ..) in real LHC environment

- Maybe first physics measurements (minimum-bias, underlying event, QCD jets, ...)?

- Observe a few $\mathrm{W} \rightarrow \mathrm{l} v, \mathrm{Y} \rightarrow \mu \mu, \mathrm{J} / \psi \rightarrow \mu \mu$ ? 
$1 \mathrm{fb}^{-1}\left(100 \mathrm{pb}^{-1}\right) \equiv 6$ months (few days) at $L=10^{32} \mathrm{~cm}^{-2} \mathrm{~s}^{-1}$ with $50 \%$ data-taking efficiency

$\rightarrow$ may collect $a \mathrm{few} \mathrm{fb}^{-1}$ per experiment by end 2008

\begin{tabular}{|l|c|l|}
\hline Channels (examples ...) & $\begin{array}{c}\text { Events to tape for } 100 \mathrm{pb}^{-1} \\
\text { (per expt: ATLAS, CMS) }\end{array}$ & $\begin{array}{l}\text { Total statistics from } \\
\text { some of previous Colliders }\end{array}$ \\
\hline$W \rightarrow \mu v$ & $\sim 10^{6}$ & $\sim 10^{4}$ LEP, $\sim 10^{6}$ Tevatron \\
$\mathrm{W} \rightarrow \mu \mu$ & $\sim 10^{6}$ LEP, $\sim 10^{5}$ Tevatron \\
$\dagger+\rightarrow W b \mathrm{~W} b \rightarrow \mu v+X$ & $\sim 10^{5}$ & $\sim 10^{4}$ Tevatron \\
$Q C D$ jets $\mathrm{P}_{\mathrm{T}}>1 \mathrm{TeV}$ & $>10^{3}$ & -- \\
$\tilde{g} \tilde{g} \quad \mathrm{~m}=1 \mathrm{TeV}$ & $\sim 50$ & -- \\
\hline
\end{tabular}

With these data:

- Understand and calibrate detectors in situ using well-known physics samples e.g. $-Z \rightarrow e e, \mu \mu \quad$ tracker, ECAL, Muon chambers calibration and alignment, etc.

- t† $\rightarrow$ blv bjj jet scale from $W \rightarrow j j$, b-tag performance, etc.

- Measure SM physics at $\sqrt{s}=14 \mathrm{TeV}: \mathrm{W}, \mathrm{Z}$, tt, QCD jets ...

(also because omnipresent backgrounds to New Physics) 


\section{Experimental conditions}

-Proton-Proton collisions @ $14 \mathrm{TeV}$

- Luminosity:

- First run in 2007 at $900 \mathrm{GeV}$

- First run @ 14 TeV in 2008, luminosity increasing to reach

$\sim 10^{33} \mathrm{~cm}^{-2} \mathrm{~s}^{-1}$ "low luminosity" phase

$\Rightarrow \sim 30 \mathrm{fb}^{-1}$ between 2008 and 2010/2011

- $10^{34} \mathrm{~cm}^{-2} \mathrm{~s}^{-1}$ "high luminosity" phase

$\Rightarrow \sim 300 \mathrm{fb}^{-1}$ by $2014 / 2015$

$\Rightarrow$ Pile-up: 2 (low luminosity) to 20 (high luminosity) pp interactions ("minimum bias") per bunch crossing (every $25 \mathrm{~ns}$ )

$\Rightarrow$ Trigger to go from $40 \mathrm{MHz}$ interaction rate to $\sim 200 \mathrm{~Hz}$ to disk for offline analysis 